\title{
Entropy-based implied volatility and its information content*
}

\author{
Xiao Xiao ${ }^{\dagger 1,2,3}$ and Chen Zhou ${ }^{\ddagger 1,2,4}$ \\ ${ }^{1}$ Erasmus School of Economics, Erasmus University Rotterdam \\ ${ }^{2}$ Tinbergen Institute, The Netherlands \\ ${ }^{3}$ Duisenburg School of Finance \\ ${ }^{4}$ De Nederlandsche Bank
}

December 5, 2016

\begin{abstract}
This paper investigates the maximum entropy method for estimating the implied volatility. Compared to existing methods the maximum entropy method has four advantages. First, it does not rely on parametric models for the risk neutral distribution. Second, it does not require a large number of options with a wide range of strike prices. Third, it allows for estimating implied skewness and kurtosis. Fourth, it allows for constructing confidence intervals around the implied volatility. We show that the maximum entropy method outperforms the existing methods if the risk neutral distribution deviates from normality. By applying this method to the S\&P500 index options, we examine the information content of the entropy-based implied volatility for forecasting future realized volatility. In some specifications, its information content is of the highest among all available volatility measures, both in terms of in-sample fit and out-of-sample predictive power. The entropy-based variance risk premium performs comparably with other alternatives in predicting future monthly market returns.
\end{abstract}

JEL Classification: C14, G13, G17

Keywords: Option pricing, risk neutral distribution, variance risk premium

*The authors thank Casper de Vries, Wolfgang Hardle, John Crosby, Matthias Fengler, Svetlana Bryzgalova, Isabel Casas, Frank Liu, Andrei Lalu, Yang Liu and Erkki Slide for helpful discussions and comments. This paper was circulated under the title "Option implied risk measures: a maximum entropy approach". Views expressed are those of the authors and do not necessarily reflect official positions of De Nederlandsche Bank.

${ }^{\dagger}$ Department of Economics, Erasmus University Rotterdam, P.O.Box 1738, 3000DR, The Netherlands. Email: xiao@ese.eur.nl.

${ }^{\ddagger}$ Economics and Research Division, De Nederlandsche Bank, 1000AB Amsterdam, The Netherlands. Email: c.zhou@dnb.nl; zhou@ese.eur.nl. 


\section{Introduction}

In financial markets, investors use options to hedge their positions against unfavorable future movements of asset prices. Consequently, option prices reflect investors' perceptions on the likelihood of having such movements. Risk measures implied by option prices can therefore be informational superior to their historical counterparts in forecasting the risk of the underlying asset. With a large literature emphasizing on the information content of option implied risk measures, little efforts have been devoted to examine whether these implied measures actually capture the characteristics of the risk neutral distribution. The situation is even more in doubt when the method of estimating option implied risk measures is based on certain parametric assumptions without empirical validation. An example reflecting this critique is the working horse methodology in practice, the Black-Scholes (BS) formula. Estimating the implied volatility by the BS formula (Black and Scholes (1973)) based on options with different strike prices results in the well-known volatility smile or smirk. This is against the uniquely defined volatility in the underlying Gaussian model. Furthermore, Neumann and Skiadopoulos (2013) show that the implied skewness calculated from S\&P500 index options is consistently negative and the implied kurtosis is always higher than three during the period from 1996 to 2010. All empirical evidence points to the fact that the risk neutral distribution observed in the financial market is inconsistent with the Gaussian assumption in the BS formula. Therefore, the Black-Scholes implied volatility (BSIV) may not capture the volatility of the risk neutral distribution accurately. This critique may apply to any parametric method for estimating the implied volatility.

In this paper, we investigate a non-parametric method, the maximum entropy (ME) method, for estimating the option implied risk measures. The estimated implied volatility using the ME method is called the entropy-based implied volatility (EBIV). We show at least four advantages of the ME method. First, the ME method does not rely on parametric models while allowing the data to determine the shape of the risk neutral distribution. Second, different from the model-free method in Britten-Jones and Neuberger (2000) and Bakshi et al. (2003), the ME method does not require a large number of options with strike prices covering a wide range. Even with limited number of options, this method can produce more 
accurate estimates than the BSIV and the model-free implied volatility (MFIV). Third, the ME method allows for calculating implied skewness and implied kurtosis. Last but not least, the ME method allows for constructing confidence intervals around the implied volatility by utilizing a nonparametric analog of likelihood ratio statistics proposed by Kitamura and Stutzer (1997).

Using non-parametric methods to extract risk measures of the risk neutral distribution has been studied extensively in the literature, in particular the so-called model-free method. This stream of literature started from the pioneer work of Britten-Jones and Neuberger (2000) and Bakshi et al. (2003), with following-up works in Dennis and Mayhew (2002), Jiang and Tian (2005), Bali and Murray (2013), Neumann and Skiadopoulos (2013), and DeMiguel et al. (2014). They show that the expected variance under the risk neutral measure can be approximated by a linear combination of European call and put option prices with strikes spanning the full range of possible values for the underlying asset at maturity. Consequently, this method makes the implied variance tradable on the market. Jiang and Tian (2005) show that the truncation error and the discretionary error of the model-free method under the stochastic volatility and random jump (SVJ) model are admissible under certain parameter specifications. However, these errors tend to be larger when the underlying distribution is more negatively skewed, when the available number of options is limited and when the market is more volatile.

This paper also contributes to the literature on testing the information content of implied risk measures. Several studies find that the implied volatility is superior to the historical volatility of the underlying asset in predicting future realized volatility; see Day and Lewis (1992), Canina and Figlewski (1993), Lamoureux and Lastrapes (1993), Christensen and Prabhala (1998), Fleming (1998), Blair et al. (2001) and Busch et al. (2011). In addition, DeMiguel et al. (2014) show that using the implied risk measures can improve the selection of mean-variance portfolios which leads to a better out-of-sample performance. We test the information content of the EBIV, and compare it with that of the BSIV and MFIV.

The ME method for extracting option implied risk measures is closely related to the principle of maximum entropy proposed in Buchen and Kelly (1996). Buchen and Kelly (1996) find that given simulated option prices at different strikes, estimating the risk neutral 
distribution by maximizing the entropy can accurately fit the true risk neutral density. In this paper, we apply this method to obtain the estimated risk neutral distribution first, and then calculate characteristics of estimated distribution, such as the EBIV, implied skewness and kurtosis. Different from Buchen and Kelly (1996), we focus on the implied risk measures rather than the full risk neutral distribution. The empirical goal of this study is to compare the estimation error and information content of the EBIV to the other alternatives such as the BSIV and MFIV. Lastly, we provide a novel methodological contribution for constructing confidence intervals around the EBIV based on Kitamura and Stutzer (1997). This study is also related to Stutzer (1996) in which options are priced using the maximum entropy method. By contrast, we conduct the reverse procedure to extract information of the risk neutral distribution from option prices.

This paper has three main contributions. First, the proposed estimators of the implied volatility, skewness and kurtosis are more accurate than their counterparts using the BS formula and the model-free method. In particular, when the risk neutral distribution exhibits heavy-tailedness and negative skewness, the EBIV is more accurate than the BSIV and MFIV. If the number of available options is reduced or the true volatility increases, the estimation error of MFIV becomes more salient while the EBIV remain robust. Second, to the best of our knowledge, this paper is the first to construct confidence intervals around implied volatility using the ME method. The coverage ratios of the constructed confidence intervals are found to be close to the confidence levels. Third, using the prices of S\&P500 index options, we provide both in-sample and out-of-sample evidence that the EBIV performs better than the BSIV and MFIV in forecasting future realized volatility. In particular, the superior performance of the EBIV is more pronounced in high volatility regimes. In addition, the variance risk premium calculated from the EBIV performs comparably or better than that based on BSIV or MFIV in forecasting future stock returns.

The remainder of the paper proceeds as follows. Section 2 discusses the estimation of the option implied risk measures using the ME method. Section 3 compares the accuracy of different implied risk measures and shows the coverage ratios of constructed confidence intervals around the EBIV. The information content of different implied volatilities are compared in Section 4. Section 5 concludes. 


\section{The entropy-based implied volatility}

We first introduce the ME method for estimating the risk neutral distribution from option prices in Section 2.1. The implied volatility is calculated from the estimated risk neutral distribution directly. Further, we explain the procedure of constructing the confidence interval around the implied volatility in Section 2.2.

\subsection{The maximum entropy method}

The ME method is a non-parametric method for estimating the risk neutral distribution with the following intuition. The absence of arbitrage guarantees the existence of a risk neutral probability measure under which the price of any security equals to the expectation of its discounted payoffs. By considering existing options prices as constraints for the underlying risk neutral distribution, one may search for the distribution that maximizes the entropy while obeying all constraints. The optimal distribution is then regarded as the estimated risk neutral distribution. In the reminder of the paper, all probability measures refer to the risk neutral probability measure.

Let $X_{t}$ represent the gross return of a stock at the expiry time $t$ in the future. Denote $S_{0}$ as the current price of the stock. At time 0 , the value of a call option with strike price $K$ equals to the expectation of its discounted payoff at time $t$ as follows:

$$
C=\mathbb{E}\left[\max \left(S_{0} X_{t}-K, 0\right)\right] / r_{t},
$$

where $r_{t}$ is the gross risk free rate from time 0 to $t$. In a discrete-state setting, we assume that there are $n$ possible states of $X_{t}$, denoted as $X_{t 1}, \cdots, X_{t n}$, with probabilities $q_{1}, \cdots, q_{n}$

respectively. In addition, we require $q_{i}>0$ and $\sum_{i=1}^{n} q_{i}=1$. The pricing equation (1) can be rewritten as:

$$
C=\sum_{i=1}^{n} q_{i}\left(\max \left(S_{0} X_{t i}-K, 0\right)\right) / r_{t}
$$

A similar pricing equation can be correspondingly established for put options.

The number of possible states is usually much larger than the number of available op- 
tions. Consequently, the pricing equations on available options are not sufficient to uniquely determine the underlying risk neutral distribution. Buchen and Kelly (1996) show that if the pricing equations are regarded as constraints on the risk neutral distribution, by maximizing the entropy, defined as

$$
\ell_{E T}=-\sum_{i=1}^{n} q_{i} \log \left(q_{i}\right)
$$

a unique optimal distribution can be obtained. Since the entropy measures the amount of missing information, the optimal distribution is the least prejudiced distribution compatible with the given constraints. For statistical inference, there is no reason to prefer any other distribution, if the only available information is the pricing equations (Buchen and Kelly (1996)).

More specifically, suppose there are $k_{1}$ call options with strike price $K_{c}(j)$ and option price $C(j), j=1, \cdots, k_{1}$. In addition, there are $k_{2}$ put options with strike price $K_{p}(j)$ and option price $P(j), j=1, \cdots, k_{2}$. Then the constraints based on the call and put options are:

$$
\begin{aligned}
C(j) & =\sum_{i=1}^{n} q_{i}\left(\max \left(S_{0} X_{t i}-K_{c}(j), 0\right)\right) / r_{t}, j=1, \ldots, k_{1} \\
P(j) & =\sum_{i=1}^{n} q_{i}\left(\max \left(K_{p}(j)-S_{0} X_{t i}, 0\right)\right) / r_{t}, j=1, \ldots, k_{2} \\
S_{0} & =\sum_{i=1}^{n} q_{i} S_{0} X_{t i} / r_{t} \\
\sum_{i=1}^{n} q_{i} & =1, q_{i}>0
\end{aligned}
$$

To present the constraints in a concise manner, we express the $k_{1}+k_{2}+1$ constraints in equations (2), (3) and (4) as:

$$
\sum_{i=1}^{n} q_{i} g_{j}\left(X_{t i}\right)=0, j=1, \ldots, k
$$

where $k=k_{1}+k_{2}+1$. The constrained optimization problem is to maximize the entropy $\ell_{E T}$ with the $k+1$ constraints. 
The Lagrange function associated with the constrained optimization problem is:

$$
\mathcal{L}=\sum_{i=1}^{n} q_{i} \log \left(q_{i}\right)+\gamma\left(\sum_{i=1}^{n} q_{i}-1\right)+\lambda^{\prime}\left(\sum_{i=1}^{n} q_{i} g\left(X_{t i}\right)\right)
$$

where $\gamma \in \mathbb{R}$ and $\lambda \in \mathbb{R}^{m}$ are the Lagrange multipliers, $g\left(X_{t i}\right)=\left(g_{1}\left(X_{t i}\right), \ldots, g_{k}\left(X_{t i}\right)\right)^{T}$. The first order conditions for $\mathcal{L}$ are solved by:

$$
\begin{aligned}
\hat{q}_{i} & =\frac{\exp \left(\hat{\lambda}^{\prime} g\left(X_{t i}\right)\right)}{\sum_{i=1}^{n} \exp \left(\hat{\lambda}^{\prime} g\left(X_{t i}\right)\right)}, i=1, \ldots, n, \\
\left(\hat{\lambda}_{1}, \ldots, \hat{\lambda}_{k}\right) & =\arg \min \sum_{i=1}^{n} \exp \left(\lambda^{\prime} g\left(X_{t i}\right)\right),
\end{aligned}
$$

where $\lambda_{j}$ is the Lagrange multiplier of the $j$ th constraint in equation (5). Notice that the estimated $\hat{q}_{i}$ is presented as a function of the Lagrange multipliers which are uniquely solved from minimizing a strictly convex function.

After estimating the risk neutral probabilities associated to the predetermined states, the EBIV is calculated as:

$$
E B I V=V^{Q}=\sqrt{\sum_{i=1}^{n} \hat{q}_{i}\left(\log \left(X_{t i}\right)-\mu^{Q}\right)^{2}}, \mu^{Q}=\sum_{i=1}^{n} \hat{q}_{i} \log \left(X_{t i}\right)
$$

We choose to calculate the EBIV of the continuously compounded returns rather than the discrete return in order to compare it later with the BSIV, because the BSIV is also based on the continuously compounded return.

In the literature, the entropy is also named as the Kullback-Leibler divergence measure, which is a member of the Cressie-Read divergence family. In fact, taking any member in the Cressie-Read divergence family as the objective function results in a non-parametric method for estimating a probability distribution under given constraints. A notable example of such a method is the so-called empirical likelihood (EL) method, in which the log likelihood function is considered as the objective function. However, there are at least two reasons why the ME method is preferred over the EL method. Empirically, the ME method provides a robust performance with respect to the variation in the possible states. Regardless whether we 
simulate states from a certain distribution, or enforce a series of equally distanced values as states, the estimated risk neutral distribution remains robust as long as the chosen states cover the range of the strike prices. On the contrary, the result following the EL method may change substantially once varying the choice of the states ${ }^{1}$. Theoretically, Theorem 1 in Schennach (2007) shows that the EL method suffers from a dramatic degradation of its asymptotic properties under even the slightest amount of misspecification.

\subsection{Constructing the confidence interval around the EBIV}

An important feature of the ME method is that it facilitates the construction of a confidence interval around the EBIV. The procedure of constructing the confidence interval follows an intuition similar to hypothesis testing. Roughly speaking, by considering the null hypothesis that the implied volatility equals to a certain value around the point estimate, one may perform a likelihood ratio with confidence level $\alpha$. Such a hypothesis would be rejected for values that are far off the point estimate. Conversely, values that are not rejected will form the confidence interval at the confidence level $1-\alpha$. A rigorous description of this idea is given as follows.

First, given the level of the mean $\mu_{Q}$, consider a hypothesis testing problem as $H_{0}$ : $V^{Q}=V_{0}^{Q}$, where $V_{0}^{Q}$ is a given level of volatility to be tested. Kitamura and Stutzer (1997) proposed the following likelihood ratio testing statistics:

$$
L R_{T}=2 n\left[\log M\left(V_{0}^{Q}\right)-\log M\left(\hat{V}^{Q}\right)\right]
$$

The two terms $M\left(V_{0}^{Q}\right)$ and $M\left(\hat{V}^{Q}\right)$ are defined as follows. The term $M\left(\hat{V}^{Q}\right)=\frac{1}{n} \sum_{i=1}^{n} \exp \left(\hat{\lambda}^{\prime} g\left(X_{t i}\right)\right)$ is the minimized value of the function (7) under the $k$ constraints in (5). The term $M\left(V_{0}^{Q}\right)=$ $\frac{1}{n} \sum_{i=1}^{n} \exp \left(\tilde{\lambda}^{\prime} g\left(X_{t i}\right)+\tilde{\lambda}_{k+1} g_{k+1}\left(X_{t i}\right)\right)$ is the minimized value of a different optimization problem

$$
\left(\tilde{\lambda}_{1}, \ldots, \tilde{\lambda}_{k}, \tilde{\lambda}_{k+1}\right)=\arg \min \sum_{i=1}^{n} \exp \left(\lambda^{\prime} g\left(X_{i}\right)+\lambda_{k+1} g_{k+1}\left(X_{i}\right)\right)
$$

\footnotetext{
${ }^{1}$ Simulation results on the comparison of the two methods are upon request.
} 
with the initial $k$ constraints in $(5)$ and an additional $(k+1)$-th constraint

$$
\sum_{i=1}^{n} q_{i} g_{k+1}\left(X_{t i}\right)=0
$$

where $g_{k+1}\left(X_{t i}\right)=\left(X_{t i}-\mu_{Q}\right)^{2}-\left(V_{0}^{Q}\right)^{2}$.

It is shown that under the null hypothesis, $L R_{T} \stackrel{d}{\rightarrow} \chi_{1}^{2}$ as $n \rightarrow \infty$. Consequently, one may vary the value of $V_{0}^{Q}$ around the estimated implied volatility $\hat{V}^{Q}$ and search for the values for which the null is not rejected under a given confidence level $\alpha$. Since $L R_{T}$ is increasing for $V_{0}^{Q}>\hat{V}^{Q}$ and decreasing for $V_{0}^{Q}<\hat{V}^{Q}$, there must exist two values $V_{L}^{Q}$ and $V_{H}^{Q}$ such that $H_{0}$ is rejected for $V_{0}^{Q}<V_{L}^{Q}$ and for $V_{0}^{Q}>V_{H}^{Q}$ while $H_{0}$ is not rejected for $V_{0}^{Q} \in\left[V_{L}^{Q}, V_{H}^{Q}\right]$. Then the interval $\left[V_{L}^{Q}, V_{H}^{Q}\right]$ is regarded as the confidence interval of $V^{Q}$ with the given confidence level $\alpha$. Obviously, we have that $\hat{V}^{Q} \in\left[V_{L}^{Q}, V_{H}^{Q}\right]$.

In this procedure, we assume that the mean of the continuous compounded return $\mu^{Q}$ is fixed when varying the constraint based on $V_{0}^{Q}$. Such an assumption is partially supported by the fact that the mean of the discrete return is fixed provided that both at-the-money call and put option prices are available. According to the put-call parity, the mean of the discrete stock return is derived as:

$$
\sum_{i=1}^{n} q_{i} X_{T i}=\frac{\left(C_{a t m}-P_{a t m}\right) r_{t}+1}{S_{0}}
$$

where $C_{a t m}$ and $P_{a t m}$ are at-the-money call and put option prices. Approximately, we regard the mean of the continuously compounded return as fixed at $\sum_{i=1}^{n} \hat{q}_{i} \log \left(X_{T i}\right)$ when we vary the value of $V_{0}^{Q}$. In the simulation, we do observe that the means of the discrete returns and the continuously compounded returns are close with the difference at a negligible magnitude.

\section{The performance of the EBIV: a numerical study}

In this section, we compare the performance of the ME method, the model-free method, and the Black-Scholes (BS) model for backing out implied volatility from option prices. In Section 3.1, we layout the technical details on how we use the BS model and the model- 
free method. The data generating process used for the simulation study is given in Section 3.2. Finally, in Section 3.3, we demonstrate that the ME method is more accurate than the other two methods when there are less number of options available and when the underlying distribution is heavy-tailed with non-zero skewness.

\subsection{The BS and model-free methods for backing out implied volatility}

The most conventional method for backing out implied volatility from option prices is to use the BS model. We first calculate the implied volatilities from all available option prices, and then take the average as the estimate of the implied volatility, denoted as the BSIV.

When the underlying return distribution deviates from log-normal, taking the average of the BS implied volatilities may not be an efficient way to aggregate information across different strike prices. Using the results in Bakshi and Madan (2000) that any payoff can be spanned and priced using an explicit positioning across option strikes, Bakshi et al. (2003) provide the explicit formula for replicating the quadratic, cubic and quartic contract. It is derived entirely from no-arbitrage conditions and can be considered as a linear combination of European call and put option prices with strikes spanning the full range of possible values for the underlying asset at maturity. In that sense, the construction of the volatility measure is of a model-free manner. The model-free implied volatility (MFIV) is defined as follows:

$$
\begin{aligned}
M F I V & =\sqrt{e^{r T} V-\mu^{2}}, \\
V & =\int_{S_{0}}^{\infty} \frac{2\left(1-\ln \left[\frac{K}{S_{0}}\right]\right)}{K^{2}} C(K, T) d K+\int_{0}^{S_{0}} \frac{2\left(1+\ln \left[\frac{S_{0}}{K}\right]\right)}{K^{2}} P(K, T) d K,
\end{aligned}
$$

where $C(K, T)(P(K, T))$ is the call (put) option price with strike price $K$ and maturity $T$ and $\mu$ is the mean of the risk neutral return, which can also be replicated by an option portfolio. $V$ is defined as $V=E^{Q}\left[e^{-r T} R_{T}^{2}\right]$. The details for calculating $\mu$ are given in Appendix 6.1. In a discrete setting, the term $V$ can be approximated as:

$$
V \approx \sum_{i=1}^{m} \frac{2\left(1-\ln \left[\frac{K_{i}}{S_{0}}\right]\right)}{K_{i}^{2}} C\left(K_{i}, T\right)\left(K_{i}-K_{i+1}\right)+\sum_{j=m+1}^{n} \frac{2\left(1+\ln \left[\frac{S_{0}}{K_{j}}\right]\right)}{K_{j}^{2}} P\left(K_{j}, T\right)\left(K_{j}-K_{j+1}\right)
$$

where $R_{T}=\ln \left[S_{T}\right]-\ln \left[S_{0}\right], K_{1}>K_{2}>\cdots>K_{m}>S=K_{m+1}>K_{m+2}>\cdots>K_{n}>$ 
$K_{n+1}=0$ are the strike prices of the available options.

Practically, since the number of available options is limited, we apply a curve-fitting method to interpolate and extrapolate the prices of the unavailable options as follows. Available option prices are first mapped to implied volatilities using the BS model. For unavailable options with strike prices within the available range, following Bates (1991) and Jiang and Tian (2005), we use cubic spines to interpolate their implied volatilities. For unavailable options with strike prices beyond the available range, we use the end-point implied volatility as their implied volatilities. Then we use the BS model to transform the obtained implied volatilities for unavailable options back to option prices. Eventually we have the option prices with moneyness ranging from 0.35 to 1.65 with an interval 0.002 . All the prices of these options are used for calculating the MFIV.

Note that there are different definitions of the model-free implied volatility. The VIX index, disseminated by the by the Chicago Board of Options Exchange (CBOE), is constructed in accordance with Britten-Jones and Neuberger (2000). The VIX is defined in the following way:

$$
V I X^{2}=E^{Q}\left[\int_{0}^{T}\left(\frac{d S_{t}}{S_{t}}\right)^{2} d t\right]
$$

The definition is not completely consistent with the definition of the EBIV. Hence, in the numerical analysis, we compare the performance of EBIV with the MFIV, which definition is consistent with the definition of EBIV.

\subsection{The underlying risk neutral distributions in the numerical study}

We consider four data generating processes to generate the underlying continuously compound returns. We start by assuming that stock price follows a geometric Brownian motion under the risk neutral measure:

$$
d S_{t}=r S_{t} d t+\sigma S_{t} d w_{t},
$$


where $S_{t}$ is the stock price at time $t, r$ is the risk-free rate, $\sigma$ is the constant instantaneous volatility of the process and $d w_{t}$ is the increment in a standard Wiener process. Throughout the section, we employ an annual risk-free rate $r$ at 5\%, an annual volatility $\sigma$ at $20 \%$ (or $40 \%$ ) and the initial stock price $S_{0}$ at 100 . Under this model, the risk neutral distribution of the continuously compounded T-year returns $\ln \left(R_{T}\right)$ is normally distributed:

$$
\ln \left(R_{T}\right) \sim N\left(\left(r-\frac{1}{2} \sigma^{2}\right) T, \sigma^{2} T\right)
$$

The mean of the risk neutral distribution, $\left(r-\frac{1}{2} \sigma^{2}\right) T$, ensures that the expectation of $R_{T}$ is $e^{r T}$ under the risk neutral measure. Note that the BS model and the model-free method are derived based on this assumption, these two methods should provide accurate estimates for the implied volatility in this case.

Next, we consider distributions deviating from the normal distribution, in particular, the Student-t and the skewed Student-t distributions. More specifically, the continuously compound return is given as

$$
\ln \left(R_{T}\right) \sim\left(r-\frac{1}{2} \sigma^{2}\right) T+\sigma \sqrt{T} \epsilon
$$

For the random term $\epsilon$, we first employ the standardized Student-t distribution with degree of freedom 5, and then the standardized skewed Student-t distribution $(\operatorname{skewt}(\eta, \lambda))$ proposed in Hansen (1994). The skewed Student-t distribution has mean 0, variance 1, a degree of freedom $\eta$ and skewness parameter $\lambda$. We use two sets of parameters: $\eta=5, \lambda=-0.3$ and $\eta=5, \lambda=-0.7$. The latter is more negatively skewed than the former.

Notice that although the mean return is comparable with that in (8), the pricing equation, $E R_{T}=e^{r T}$, does not hold if $\epsilon$ follows the non-normal distributions, though it remains approximately true.

\subsection{Results}

Based on the risk neutral distributions specified in Section 3.2, we calculate the call and put option prices using numerical integration for several moneyness with one month to expiration. 
Results for other expiration horizons are provided in the robustness check in Section 3.4. The call and put option prices with strike price $K$ and maturity $T$ are calculated by numerical integration:

$$
\begin{aligned}
C(K, T) & =\int_{K / S_{0}}^{\infty}\left(S_{0} R_{T}-K\right) f\left(R_{T}\right) d R_{T} / r_{T} \\
P(K, T) & =\int_{0}^{K / S_{0}}\left(K-S_{0} R_{T}\right) f\left(R_{T}\right) d R_{T} / r_{T}
\end{aligned}
$$

where $f\left(R_{T}\right)$ is the density function of $R_{T}, r_{T}$ is the risk-free rate that used to discount payoff of the options.

Following Bakshi et al. (2003), we only consider out-of-the-money (OTM) options and atthe-money (ATM) options because in-the-money options are less traded in the option market, while their prices can be derived from the put-call parity under the no-arbitrage condition. Consequently, they do not provide additional information for extracting the implied volatility.

We consider different ranges of strike prices which result in different estimation accuracy. In the first case, we specify the moneyness $\left(K / S_{0}\right)$ of call options from 1 to 1.15 with equal interval 0.025 , and the moneyness of put options from 0.85 to 1 with the same interval. There are 14 options in total. In the second case, we reduce the number of available options and only consider six options: call options with moneyness 1, 1.05, 1.1 and put options with moneyness $0.95,0.975,1$. By comparing the two cases, we evaluate the performance of the three methods with different number of available options. The calculated option prices with the chosen moneynesses under different distributions are reported in Table 1.

Table 2 reports the estimated implied volatilities under different distributions using the three methods. The first row shows the true volatility of the underlying distribution and the second row shows the number of options. In the parenthesis, We report the relative improvements of the MFIV and EBIV compared to the BSIV, which are defined as $\frac{\mid X X I V-\text { TrueVolatility } \mid}{\mid B S I V-\text { TrueV olatility } \mid}$, where XXIV is either MFIV or EBIV.

From Table 2, we observe that under the normal distribution, all three methods provide accurate estimates. However, when the underlying distribution is heavy-tailed or negatively skewed, the EBIV estimates are closer to the true value than both the BSIV and MFIV. The improvement is substantial. 
Although the MFIV performs better than the BSIV under heavy-tailed or skewed distributions, the estimation error increases when the underlying distribution is more negatively skewed. However, the estimation improvement of the EBIV remains robust across different specifications. When we decrease the available number of options from 14 to 6 or increase the true volatility from 0.2 to 0.4 , the better performance of EBIV compared to MFIV becomes more evident. For example, an estimation error of the MFIV under the skewt $(5,-0.7)$ distribution with 6 options and true volatility 0.4 is even larger than that of the BSIV, while the EBIV has an estimation error as low as $31 \%$ of that of the BSIV. The unrobust performance of the MFIV may be attributed to the increase of the truncation error and extrapolation error, whereas the ME method does not suffer from such a problem.

An additional advantage of the ME method is that one may construct the confidence interval around the estimate of the implied volatility. Table 3 shows the coverage rate of the EBIV confidence interval under the four distributions with six option prices. This analysis is conducted as follows. We simulate 10000 states from the true distribution for 100 times $^{2}$. For each set of simulated states, we first calculate option prices, and then construct the confidence interval for the implied volatility. Next, we examine the confidence interval covers the true volatility, i.e. whether the true volatility falls into the constructed interval. Across the 100 simulations, we count the number of "coverage" and divide that number by 100. From Table 3 , we find that the coverage rates of the confidence interval are close to the confidence levels under all four distributions.

\subsection{Robustness and discussion}

Results from Section 3.3 show that the EBIV aggregates information in the option prices more efficiently than the MFIV, when the underlying distribution exhibits heavy tail and when the number of options is limited. We now conduct robustness check to ensure the generality of our findings.

In Table 4 and Table 5, we provide the estimated implied volatilities based on option

\footnotetext{
${ }^{2}$ When simulating the states, kurtosis of the simulated sample might differ from the true value in many simulations. The reason is that sample kurtosis is very sensitive to extreme observations. If there are no extreme observations in the simulated sample, sample kurtosis is downward biased. To alleviate this bias, we only consider the simulated sample if the sample kurtosis is higher than $80 \%$ of the true kurtosis.
} 
prices with three-month and one-year maturity, respectively. In addition to the 14 or 6 options with symmetric moneyness, we consider asymmetric moneyness with 3 call options (moneyness ranging from 1 to 1.05) and 7 put options (moneyness ranging from 0.85 to 1 ), with equal interval 0.025 . This is to reflect that there are more available put options than call options traded in the market. Column 5 an 8 in Table 4 and Table 5 report the results when having asymmetric moneyness. Results in these two tables show that the EBIV still has a better estimation accuracy than the MFIV and BSIV for longer maturity options.

The second generalization is to consider more complex data generating process for the risk neutral distribution, for example, the SVJ model. Further, with such data generating process, the risk neutral distribution possesses non-trivial skewness and kurtosis. We can thus examine the performance of the ME method for estimating implied skewness and kurtosis. The SVJ model has been applied for pricing options in Bakshi et al. (1997) and for illustrating truncation errors of model-free implied volatility in Jiang and Tian (2005). The model is specified as:

$$
\begin{aligned}
\frac{d S_{t}}{S_{t}} & =\sqrt{V_{t}} d W_{t}+J_{t} d N_{t}-\mu_{J} \lambda d t, \\
d V_{t} & =\left(\theta_{v}-\kappa_{v} V_{t}\right) d t+\sigma_{v} \sqrt{V_{t}} d W_{t}^{v}, \\
d W_{t} d W_{t}^{v} & =\rho d t
\end{aligned}
$$

where $N_{t}$ follows a homogeneous Poisson process with jump intensity $\lambda$ and $\ln \left(1+J_{t}\right)$ follows a normal distribution $N\left(\ln \left(1+\mu_{J}\right)-\frac{1}{2} \sigma_{J}^{2}, \sigma_{J}^{2}\right)$. If $\lambda=0$, the model reduces to the Heston (1993) model. We choose the parameters as $\kappa_{v}=1, \sigma_{v}=0.25, \rho=0, \lambda=0.5, \theta_{v}=V_{0} \kappa_{v}$, $V_{0}=0.1854^{2}$. In addition, to evaluate the impact of the jump process, we choose two sets of parameters for the jumps: (1) $\mu_{J}=-1.75, \sigma_{J}=0.5,(2) \mu_{J}=-0.075, \sigma_{J}=2.5$, correspondingly. The volatility is 0.203 for the first set of parameters and 0.453 for the second set of parameters.

Since the unconditional return distribution are unknown here, it is necessary to conduct pre-simulations to obtain option prices and the true skewness and kurtosis. First, we simulate 21 daily returns to get one monthly return, and repeat this for 100,000 times. Second, option prices, the true volatility, skewness and kurtosis are calculated based on these simulated 
monthly return.

With the obtained option prices, we estimate the implied volatility by the BS model, the model-free method and the ME method, and compare the estimates with the true volatility. In addition, we calculate the implied skewness and kurtosis using the model-free method and the ME method. The results for options with one-month maturity are reported in Table 6: the second column for each parameter set reports the simulated moments and the column 3-5 reports the implied moments estimated from different methods. The results show that the ME method gives the most accurate estimation in all cases, and is especially robust when the unconditional distribution has higher volatility, more negative skewness and higher kurtosis.

Lastly, we check the robustness of the ME method by focusing on its fundamental step: the estimated risk neutral distribution. For the original four data generate processes, we compare the sample of the simulated distribution (blue bars) and the estimated density produced by the ME method (red lines) in Figure 1. More specifically, the estimated risk neutral densities in these figures are estimated from 14 options with one year maturity. The figures show that the risk neutral density estimated by the ME method matches the true density in all four cases. Option prices with different moneynesses essentially provide information on different parts of the distribution.

To conclude, the ME method provides more accurate estimates of option implied volatility than the BS model and the model-free method. Our main findings are robust to the choice of different number of options, maturities and data generating process. In addition, the ME method can also be applied for other higher moments of the risk neutral distribution, because it provides an accurate estimation for the risk neutral distribution.

\section{The information content of EBIV}

In this section, we conduct an empirical analysis to explore the information content of the EBIV using the S\&P500 index option traded in the Chicago Board Options Exchange (CBOE). We first review the basic statistical properties of the various volatility measures and then investigate their relative performance as predictors for the subsequent realized volatilities of the underlying S\&P500 index. Further, we analyze the forecasting power of the 
variance risk premia derived from different implied volatilities on the subsequent returns of the S\&P500 index.

The volatility measures we include in the empirical analysis are: lagged realized volatility (RV), Black-Scholes implied volatility (BSIV), VIX provided by CBOE, model-free implied volatility (MFIV) and entropy-based implied volatility (EBIV). We include both the VIX and the MFIV because the construction methodologies of these two measures are different as illustrated in Section 3. In addition, the VIX measure is not truly model free because the derivation of this measure involves assumptions of the underlying process. While Jiang and Tian (2005) and Carr and Wu (2009)) argue that jumps are unlikely to create sizable biases in VIX, this view has been revised in Carr et al. (2012), Andersen et al. (2015) and Martin (Forthcoming). The problem is that price jumps induce a discrepancy between the fair value of future cumulative squared returns and $V I X^{2}$, even in the continuous sampling limit. The MFIV is more consistent to the model-free manner because the method does not depend on distribution assumption on the underlying process.

\subsection{Data}

Our sample period covers from January 1996 to August 2014. We get the S\&P500 index price data from The Center for Research in Security Prices database. We obtain the S\&P500 index options data from the Ivy DB database of OptionMetrics. Continuously-compounded zero-coupon interest rates are also obtained from OptionMetrics as a proxy for the risk-free rate. From the CBOE, we get daily levels of the newly calculated VIX index ${ }^{3}$ and match them with the trading days on which options with one month expiration are traded.

Our analysis is conducted based on call and put options quoted on the S\&P500 index with 30 days expiration. We choose one-month maturity because the options with one month to expire are more actively traded than with other maturities. From January 1996 to February 2007, in each month, there is only one day on which options with 30 days expiration are traded. From March 2007, there are several such days in each month. To avoid the overlapping problem described in Christensen and Prabhala (1998), Christensen et al. (2001)

\footnotetext{
${ }^{3}$ Although the CBOE changed the methodology for calculating the VIX in September 2003, they have backdated the new index using the historical option prices.
} 
and Jiang and Tian (2005), we select one date in each month from 2006 to 2014, such that the time intervals between any two adjacent dates are the closest to 30 days. With this procedure, there are 222 selected dates in total. Midpoints of the bid-ask spread are used as the option prices instead of the actual trade prices. This follows Jackwerth (2000) who demonstrates that measurement of risk neutral distribution is not sensitive to the existence of spreads.

Table 7 presents the descriptive statistics of the out-of-the-money call and put options. We apply several filters to select the options. First, option quotes less than $3 / 8$ are excluded from the sample. Such low prices may not reflect the true option value due to proximity to tick size. Second, options with zero open interest are excluded from the sample. Third, following Aït-Sahalia and Lo (1998) and Bakshi et al. (2003), we exclude in-the-money options, because they have less liquidity than out-of-the-money options.

For BSIV, we calculate the mean of the Black-Scholes implied volatility using all available option prices after the filtering procedure.

We use all available option prices with moneyness between 0.85 to 1.15 to calculate the MFIV. We interpolate and extrapolate the prices of the unavailable options using the method discussed in Section 3.1. Eventually we have option prices with moneyness ranging from 0.35 to 1.65. All the prices of these options are used for calculating the MFIV.

By contrast, we do not use all option prices in the ME method. To calculate the EBIV, we select options with moneyness that are closest to the moneyness ranging from 0.85 to 1.15 with equal interval 0.025 . The reason is that with more option prices as constraints, the ME method may run into numerical difficulties as follows. If the covariance matrix of the constraints in equation $(5), \operatorname{cov}\left(g_{i}\left(X_{t}\right), g_{j}\left(X_{t}\right)\right)$, is close to singular, then the numerical solution for the Lagrange multipliers becomes unstable (Buchen and Kelly (1996)).

On each selected trading day, we also calculate the historical realized volatility (RV) in the previous month. Following Christensen and Prabhala (1998), we adopt the realized volatility over the 30 calendar days proceeding the current observation dates as the lagged realized volatility $R V_{t}$. It is computed as the sample standard deviation of the daily index 
returns:

$$
R V_{t}=\sqrt{\frac{1}{30} \sum_{i=1}^{30}\left(r_{t, i}-\bar{r}_{t}\right)^{2}}
$$

where $\bar{r}_{t}=\frac{1}{30} \sum_{k=1}^{30} r_{t, k}$, and $r_{t, i}, i=1, \ldots, 30$, are the $\log$ index returns on the 30 days preceding to the selected trading day $t$. All of the volatility measures are expressed in annual terms to facilitate interpretation.

\subsection{Descriptive statistics of different volatility measures}

Table 8 reports descriptive statistics of the five measures of volatility: RV, VIX, BSIV, MFIV and EBIV. Table 9 shows the correlation matrix of these measures. We first observe that the mean of the four implied volatility measures, VIX, BSIV, MFIV and EBIV, are comparable. All of them exceed the mean of the realized volatility measure RV by about $24 \%$, which is in line with the positive volatility risk premium. Second, the four implied volatility measures are highly correlated, with all correlation coefficients above 0.99. VIX is more correlated with MFIV and EBIV than with BSIV. This may be a consequence of the fact that the three shares the same nonparametric feature by construction.

In Figure 2, we plot the estimated MFIV and EBIV from 1996 to 2014. Figure 2a shows their strong comovement during the period. From Figure $2 b$, we observe that in most of the time, the differences between the EBIV and the MFIV are negative and small. Occasionally, the differences can be positive and large. This is consistent with the results in Table 4 and 5 : when the number of the put options is more than the number of call options, the MFIV may overestimate the true volatility under the low volatility regime $(\sigma=20 \%)$. Conversely, the underestimation occurs under the higher volatility regime $(\sigma=40 \%)$. To further illustrate this observation, Figure 2c plots the spread between the EBIV and the MFIV against the BSIV. By regarding the BSIV as an indication of high and low volatility regime, this scatter plot further demonstrates that in a higher volatility regime, the spread is also higher. We attribute this phenomenon to the fact that the model-free method produces less accurate estimates of implied volatility when the market condition becomes more volatile.

Figure 3a presents the confidence interval for the estimated EBIV and Figure 3b shows 
the length of the confidence interval over time. Lastly, in Figure 4, we provide the estimated risk neutral distributions on four example dates using the ME method. From the figures, we observe that the estimated risk neutral distributions may differ across different market environments.

\subsection{Forecasting the stock market volatility}

Prior research has extensively analysed the information content of the BSIV on predicting the future realized volatility. In particular, recent studies seem to agree on the informational superiority of the BSIV compared to historical volatility. In this paper, we assess the predictive power of the EBIV, and compare it to the other implied volatility measures.

To nest previous research within our framework, we use five competing volatility measures: $R V_{t}, B S I V_{t}, V I X_{t}, M F I V_{t}$ and $E B I V_{t}$ to forecast the realized volatility in the next period $R V_{t+1}$. To explore the predictive ability of the implied volatility measures, we first include each of them within an in-sample regression separately. We run the following regression

$$
R V_{t+1}=\alpha_{i}+\beta_{i} X_{i, t}+\epsilon_{i, t+1}
$$

with different predictors $X_{i, t} \in I=\left\{R V_{t}, B S I V_{t}, V I X_{t}, M F I V_{t}, E B I V_{t}\right\}$. Adjusted $R^{2}$ of these regressions captures the proportion of total variation in the ex-post realized volatility explained by the predictors.

We also employ encompassing regressions to investigate whether EBIV has additional predictive information compared with other volatility measures. To mitigate the multicollinearity problem, we first regress $E B I V_{t}$ on the other volatility measures and get the error term. Then, we run the bivariate regression of the volatility measures and the error term from the first step. The regressions are specified as follows:

$$
\begin{aligned}
& \text { First Step: } E B I V_{t}=\alpha_{E B I V, i}+\beta_{E B I V, i} X_{i, t}+\epsilon_{E B I V, X_{i}, t} \\
& \text { Second Step: } R V_{t+1}=\alpha_{i}+\beta_{1, i} X_{i, t}+\beta_{2, i} \epsilon_{E B I V, X_{i}, t}+\epsilon_{t}, i \neq 5,
\end{aligned}
$$

where $X_{i, t}$ an element from $I$, which is different from $E B I V_{t}$. 
Furthermore, we investigate whether other volatility measures have additional information content in predicting the future realized volatility, compared with $E B I V_{t}$. Similarly, we estimate the following two-step regressions:

$$
\begin{gathered}
\text { First Step: } X_{i, t}=\alpha_{i, E B I V}+\beta_{i, E B I V} E B I V_{i, t}+\epsilon_{X_{i}, E B I V, t} \\
\text { Second Step: } R V_{t+1}=\alpha_{i}+\beta_{1, i} E B I V_{i, t}+\beta_{2, i} \epsilon_{X_{i}, E B I V, t}+\epsilon_{t}, i \neq 5,
\end{gathered}
$$

Table 10 summarizes the results of both univariate and the second-step encompassing regressions for the realized volatility in the next month. First, from the estimation results of univariate regressions in Panel A Table 10, we observe that the adjusted $R^{2}$ is the highest when using the EBIV compared to other implied volatility measures. Second, in the bivariate regressions with volatility measures and the uncorrelated EBIV residuals, the coefficients of the EBIV residual term are all statistically significant at $10 \%$ in Panel B. This indicates that the EBIV can explain some variations in the future realized volatility that other volatility measures cannot explain. Third, when we include EBIV in the predictive regression, none of the additional information in RV, BSIV, VIX and MFIV is statistically significant at $10 \%$. In Panel $\mathrm{C}$, the coefficients of EBIV are all significant at 1\%, while the error terms of the other volatility measures regressing on EBIV are not significant. The results indicate that the EBIV plays a dominant role in explaining the variations of the future realized volatility. In summary, the evidence suggests that, among all the implied volatility measures, the EBIV explains the most variation in the next month realized volatility with the highest in-sample fit. It is also notable that even if the MFIV uses more options as inputs, its information content does not overweight that of EBIV.

We then turn to the out-of-sample evidence reported Table 11. We use moving window of 100 observations preceding to the period to be forecasted as the estimation window in the regression. Consequently, the remaining 122 months are the forecasting period. We use the mean squared forecasting error (MSFE) as the overall measure of forecasting accuracy. We choose MSFE for two reasons. First, it is the most widely used loss function in the volatility forecasting literature. Second, Patton (2011) shows that it is one of the loss function that yields inference that is invariant to the choice of units of measurement. If we denote $\widehat{R V}_{t}$ as 
a forecast for $R V_{t}$, the MFSE is formally defined as,

$$
M F S E=\frac{\sum_{t=101}^{222}\left(\widehat{R V}_{t}-R V_{t}\right)^{2}}{122}
$$

To compare the out-of-sample performance of the competing implied volatility measures, we compute the Diebold and Mariano (1995) and West (1996) (DMW) statistic for testing the null of equal predictive ability) $\left(M F S E_{j}=M F S E_{i}\right)$ against the alternative that the competing measure has a lower MSFE than the baseline measure $\left(M F S E_{j}>M F S E_{i}\right)$. In Panel A Table 11, we report the full-sample MFSE ratio and DMW statistics in the parenthesis, The MFSE ratio is defined as follows:

$$
\operatorname{MFSE}\left(X_{i}, X_{j}\right)=\frac{\operatorname{MFSE}\left(X_{j}\right)}{\operatorname{MFSE}\left(X_{i}\right)}
$$

where $X_{i}$ represent the implied volatility measures on the first column and $X_{j}$ represent those on the first row. Panel A shows that in the full sample, the rank of out-of-sample MFSE is RV, BSIV, VIX, MFIV and EBIV from the largest to the smallest. In terms of the significance of DMW statistics, we can see that the out-of-sample performance of EBIV is significantly better than VIX and MFIV at 5\%. For other pairs of competing implied volatility measures, we do not observe any statistical significance.

Since the EBIV is particularly accurate in backing out the implied volatility during high volatility periods, we divide the monthly forecast of future volatility into three subsamples by sorting the BSIV preceding to the forecasted month in ascending order. Panel B, C and $\mathrm{D}$ in Table 11 report the results in these three subperiods, indicated by the "Low volatility period", "Medium volatility period" and "high volatility period" columns. First, in the low volatility period, all implied volatility measures significantly outperform the lagged realized volatility measure (RV) at 5\%. All the model free implied volatility measures outperform the BSIV at $10 \%$. MFIV has the best performance among all competing measures. Second, in the medium volatility period, VIX has the best out-of-sample predictive power. Third, in the high volatility regime, the forecasting error of EBIV is smaller than the other four implied volatility measures, but EBIV only significantly outperforms VIX and MFIV.

We also examine whether the out-of-sample performance has significant improvement 
when we include additional variables in the predictive regression. We calculate two MSFE ratios: $\operatorname{MFSE}\left(X, X, \epsilon_{E B I V, X}\right)$ and $M F S E\left(E B I V, E B I V, \epsilon_{X, E B I V}\right)$. The first one is used to investigate the out-of-sample performance after we add the uncorrelated error term of EBIV in the univariate predictive regression of the other implied volatility measures. The second one aims at analyzing whether there is incremental out-of-sample performance when we add the other implied volatility measures to the univariate regression of the uncorrelated EBIV error term. To assess the relative predictive power of two nested models, we use the MSFEadjusted statistic suggested in Clark and West (2007). We summarize the MSFE ratio and the DMW statistics of the encompassing models in Table 12. The table shows that the outof-sample performance of the univariate model for RV, BSIV and VIX improved significantly after we add the uncorrelated EBIV term in the model. However, adding the uncorrelated EBIV term decreases the out-of-sample performance of MFIV. The table also shows that the out-of-sample prediction performance of EBIV cannot be improved by including information in other implied volatility measures.

As a further robustness check, we provide in-sample and out-of-sample results based on the log-level of the realized volatilities in Table 13 and Table 14. In this log volatility specification, we find that the EBIV and MFIV have the highest in-sample adjusted $R^{2}$ among all the implied volatility measures. The additional information in EBIV has significant predictive power that RV and BSIV do not have, but it is not significant in the regression of VIX and MFIV. When we regress the EBIV and the uncorrelated other implied volatility measures, none of these measures are significant. In addition, in Table 15, it is robust that the smallest out-of-sample MFSE is still obtained by using the EBIV in the full sample. However, it is not significantly better than MFIV in the full sample and in the high volatility period.

Finally, we conduct a robustness check by using a broader choice of strike prices, i.e. moneyness ranging from 0.5 to 1.5 . The quantitative results remain valid ${ }^{4}$. An additional observation is that the adjusted $R^{2}$ when using the BSIV as a sole regressor becomes smaller when we incorporate more options. It shows that using the BS formula is not efficient for integrating information in a large number of option prices. By contrast, the adjusted $R^{2}$ of

\footnotetext{
${ }^{4}$ Regression results and out-of-sample analysis are available upon request.
} 
using the EBIV as the sole regressor increases in this case. Therefore, the ME method can better integrate the information contained in multiple option prices.

To sum up, EBIV has the smallest out-of-sample forecasting error among all the competing implied volatility measures. During the low, medium and high volatility period, the differences of the forecasting errors between EBIV and other implied volatility measures are statistically significant in terms of DMW statistics in some specifications. The extra information in EBIV significantly improves the out-of-sample performance of RV, BSIV and VIX, while the extra information in other implied volatility measures does not significantly improve the out-of-sample performance of EBIV.

\subsection{Forecasting stock market returns}

The theoretical model in Bollerslev et al. (2009) suggest that variance risk premium (VRP) may serve as a predictor for future returns. The variance risk premium is defined by the difference between the ex ante risk-neutral expectation of the future return variation over the $[t, t+1]$ time interval and the ex post realized return variation over the $[t-1, t]$ time interval: $V R P_{t}=I V_{t}-R V_{t}$. Note that we implicitly assume that $R V_{t}$ is a martingale process and $R V_{t}$ measures the expection of realized variance $E_{t}\left[R V_{t+1}\right]$. The advantage is that this VRP measure does not depend on the specification of the forecast model for the future variance. Instead, it is completely model-free. In this paper, we intend to compare the performance of VRP using different methods for backing out the implied variance. We use univariate regressions to examine the in-sample fit and out-of-sample forecasting performance.

Denote the ex-post return for month $t+1$ as $R_{t+1}$, the regressions take the form:

$$
R_{t+1}=\alpha_{i}+\beta_{i} x_{i, t}+\epsilon_{i, t+1}
$$

where $x_{i, t}$ is one of the item in $I=\left\{V R P_{B S, t}, V R P_{M F, t}, V R P_{E B, t}\right\}, V R P_{B S, t}=B S I V_{t}^{2}-$ $R V_{t}^{2}$ and $V R P_{M F, t}$ and $V R P_{E B, t}$ are calculated based on $M F I V_{t}$ and $E B I V_{t}$ in a similar way.

Table 16 reports the results of predicting future monthly returns. In all regressions, the estimated slope coefficients associated with the VRP measures are significant at 5\% 
confidence level. In addition, $V R P_{E B}$ explains more variations in future monthly returns than $V R P_{B S}$ and $V R P_{M F}$ with the highest $R^{2}$ at $7.1 \%$. The out-of-sample setup is similar to that in Section 4.3. The only difference is that the variable to be forecasted here is the monthly stock return $R_{t}$ instead of the realized volatility $R V_{t}$. In the out-of-sample results, $V R P_{E B}$ performs as good as $V R P_{M F}$. The $V R P_{E B}$ and the $V R P_{M F}$ both perform better than $V R P_{B S}$ and they forecast more accurately in the Medium and High volatility regimes. The differences between the MFSE of different VRP measures are not statistically significant in terms of the DMW statistics.

Our empirical results point to the direction that the EBIV performs at least at a comparable level with the MFIV in different specifications. In many cases, its information content is of the highest among all available volatility measures, both in terms of in-sample fit and out-of-sample predictive power.

\section{Conclusion}

This paper provides the first comprehensive investigation on the option implied volatility estimated by the ME method. The ME method extracts the risk neutral distribution of an asset, given a set of option prices at different strikes. The EBIV is then calculated based on the estimated risk neutral distribution. Compared to parametric methods such as the BS model, the ME method does not depend on any parametric assumption. Compared to the MFIV, proposed by Bakshi et al. (2003), the ME method does not require many options with strike prices spanning the full range of possible values for the underlying asset at expiry. Therefore, the ME method combines the advantages in the model-free and the parametric methods: on the one hand, it aggregates information in multiple options with different strikes efficiently; on the other hand, it produces accurate estimates even if the number of options is limited. Lastly, it allows for constructing confidence interval around the estimated implied volatility thanks to a nonparametric analog of the likelihood ratio test.

With numerical examples, we show that the EBIV has a lower estimation error than the BSIV and the MFIV, particularly when the underlying distribution exhibits heavy tail and non-zero skewness. With limited number of available options or under high volatility level, 
the accuracy of the EBIV remains robust while the estimation error of the MFIV gets higher. The confidence interval around the EBIV has a coverage ratio that is close to the correct confidence level across various numerical examples. These findings are robust to the choice of different number of options, maturities and data generating process.

We remark that the ME method also yields estimators for other higher moments of the risk neutral distribution, such as skewness and kurtosis. The estimators perform better than their counterparts when using the model-free method. A potential explanation is that the ME method provides an accurate estimation for the risk neutral distribution.

Using the S\&P500 index options, we empirically test the information content of the EBIV in predicting future monthly realized volatilities and index returns. Our empirical results point to the direction that the EBIV performs at least at a comparable level with the MFIV in different specifications. In many cases, its information content is of the highest among all available volatility measures, both in terms of in-sample fit and out-of-sample predictive power.

A potential drawback of the ME method is that the tail region of the estimated risk neutral distribution largely depends on the options with the highest and lowest strike prices. Given limited number of available options, the estimated density can be less accurate for that part. This may be the reason why estimating implied skewness and kurtosis is less accurate than implied volatility. Improving the estimation of the tail region of the risk neutral density and the option implied skewness and kurtosis is left for future research. 


\section{Appendix}

\subsection{Calculation of the Model-free implied moments}

The calculation of the model-free option implied moments follows from Bakshi et al. (2003). Let the $t$-period continuous compounded return be given by: $R_{t}=\ln \left[S_{t}\right]-\ln \left[S_{0}\right]$. The fair values of the mean, volatility, cubic and quartic contract at time 0 are defined as:

$M(0, t)=E\left[e^{-r t} R_{t}\right], \quad V(0, t)=E\left[e^{-r t} R_{t}^{2}\right], \quad W(0, t)=E\left[e^{-r t} R_{t}^{3}\right]$, and $X(0, t)=E\left[e^{-r t} R_{t}^{4}\right]$

To simplify the notations, we ignore the time period information in the parenthesis in the following equations, for instance $V=V(0, t)$. Further, under the risk neutral measure, the values $M, V, W$ and $X$ can be replicated by the option prices as,

$$
\begin{aligned}
M & =1-e^{-r t}-\frac{1}{2} V-\frac{1}{6} W-\frac{1}{24} X, \\
V & =\int_{S}^{\infty} \frac{2\left(1-\ln \left[\frac{K}{S_{0}}\right]\right)}{K^{2}} C(K, t) d K+\int_{0}^{S} \frac{2\left(1+\ln \left[\frac{S_{0}}{K}\right]\right)}{K^{2}} P(K, t) d K \\
W & =\int_{S}^{\infty} \frac{6 \ln \left[\frac{K}{S}\right]-3\left(\ln \left[\frac{K}{S_{0}}\right]\right)^{2}}{K^{2}} C(K, t) d K-\int_{0}^{S} \frac{6 \ln \left[\frac{K}{S}\right]+3\left(\ln \left[\frac{S_{0}}{K}\right]\right)^{2}}{K^{2}} P(K, t) d K, \\
X & =\int_{S}^{\infty} \frac{12\left(\ln \left[\frac{K}{S}\right]\right)^{2}-4\left(\ln \left[\frac{K}{S_{0}}\right]\right)^{3}}{K^{2}} C(K, t) d K-\int_{0}^{S} \frac{12\left(\ln \left[\frac{K}{S}\right)^{2}\right]+4\left(\ln \left[\frac{S_{0}}{K}\right]\right)^{3}}{K^{2}} P(K, t) d K .
\end{aligned}
$$

The $t$-period risk neutral return mean $\mu$, volatility $M F I V$, skewness MFIS, and kurtosis $M F I K$ are given as

$$
\begin{aligned}
\mu & =e^{r t} M, \\
M F I V & =\sqrt{e^{r t} V-\mu^{2}} \\
M F I S & =\frac{e^{r t} W-3 \mu e^{r t} V+2 \mu^{3}}{\left(e^{r t} V-\mu^{2}\right)^{3 / 2}}, \\
M F I K & =\frac{e^{r t} X-4 \mu e^{r t} W+6 e^{r t} \mu^{2} V-3 \mu^{4}}{\left(e^{r t} V-\mu^{2}\right)^{2}} .
\end{aligned}
$$


Table 1: Option prices under different risk neutral distributions

(a) Panel A: $\sigma=0.2$

\begin{tabular}{rrrrrr}
\hline & Moneyness $\left(K / S_{0}\right)$ & lognormal & Student t & Skewt1 & Skewt2 \\
\hline Call & 1.15 & 0.020 & 0.078 & 0.020 & 0.000 \\
& 1.125 & 0.057 & 0.125 & 0.038 & 0.001 \\
1.1 & 0.148 & 0.210 & 0.080 & 0.003 \\
& 1.075 & 0.349 & 0.373 & 0.188 & 0.022 \\
1.05 & 0.744 & 0.691 & 0.474 & 0.237 \\
& 1.025 & 1.435 & 1.292 & 1.146 & 1.002 \\
& 1 & 2.512 & 2.333 & 2.336 & 2.310 \\
& & & & & \\
Put & 0.85 & 0.003 & 0.029 & 0.062 & 0.093 \\
& 0.875 & 0.015 & 0.054 & 0.105 & 0.149 \\
& 0.9 & 0.061 & 0.107 & 0.184 & 0.242 \\
& 0.925 & 0.193 & 0.222 & 0.329 & 0.402 \\
& 0.95 & 0.504 & 0.469 & 0.598 & 0.675 \\
& 0.975 & 1.106 & 0.979 & 1.086 & 1.137 \\
1 & 2.096 & 1.917 & 1.922 & 1.899 \\
\hline
\end{tabular}

(b) Panel B: $\sigma=0.4$

\begin{tabular}{rrrrrr}
\hline & Moneyness $\left(K / S_{0}\right)$ & lognormal & Student t & Skewt1 & Skewt2 \\
\hline Call & 1.15 & 0.730 & 0.811 & 0.394 & 0.041 \\
& 1.125 & 1.049 & 1.063 & 0.598 & 0.131 \\
1.1 & 1.479 & 1.410 & 0.921 & 0.400 \\
& 1.075 & 2.046 & 1.882 & 1.417 & 0.947 \\
1.05 & 2.774 & 2.521 & 2.140 & 1.779 \\
& 1.025 & 3.688 & 3.368 & 3.123 & 2.886 \\
& 1 & 4.805 & 4.456 & 4.367 & 4.247 \\
& & & & & \\
Put & 0.85 & 0.353 & 0.396 & 0.580 & 0.702 \\
& 0.875 & 0.613 & 0.602 & 0.814 & 0.944 \\
& 0.9 & 1.003 & 0.913 & 1.138 & 1.266 \\
& 0.925 & 1.552 & 1.369 & 1.582 & 1.691 \\
& 0.95 & 2.289 & 2.017 & 2.180 & 2.248 \\
& 0.975 & 3.231 & 2.897 & 2.967 & 2.966 \\
1 & 4.390 & 4.036 & 3.976 & 3.879 \\
\hline
\end{tabular}

Note: This table reports call and put option prices with different moneynesses under different risk neutral distributions. Panel A reports results for $\sigma=0.2$ and Panel B reports for $\sigma=0.4$. Risk neutral distributions of the continuously compounded stock returns follow the normal, the Student-t or two skewed Student-t distributions as specified in (8) and (9). The degree of freedom of the Student-t and the two skewed Student-t distributions is 5. For the two skewed Student-t distributions, the skewness parameters are -0.3 and -0.7 . The risk-free rate is $5 \%, K$ is the strike price, $S_{0}$ is the initial stock price 100 . 
Table 2: Comparison across the three methods, one-month maturity

\begin{tabular}{crrrrr}
\hline \multirow{5}{*}{ Normal } & Volatility $\sigma$ & 0.2 & 0.2 & 0.4 & 0.4 \\
& Option No. & 14 & 6 & 14 & 6 \\
& BSIV & 0.200 & 0.200 & 0.400 & 0.400 \\
& MFIV & 0.200 & 0.200 & 0.400 & 0.400 \\
& EBIV & 0.200 & 0.202 & 0.402 & 0.413 \\
Student-t & & & & & \\
& BSIV & 0.211 & 0.192 & 0.385 & 0.373 \\
& MFIV & 0.198 & 0.195 & 0.387 & 0.374 \\
& & $(0.209)$ & $(0.628)$ & $(0.896)$ & $(0.979)$ \\
& EBIV & 0.199 & 0.196 & 0.393 & 0.393 \\
& & $(0.139)$ & $(0.466)$ & $(0.444)$ & $(0.264)$ \\
& & & & & \\
& BSIV & 0.206 & 0.192 & 0.374 & 0.368 \\
& MFIV & 0.197 & 0.197 & 0.383 & 0.366 \\
& & $(0.433)$ & $(0.342)$ & $(0.657)$ & $(1.082)$ \\
& EBIV & 0.198 & 0.196 & 0.391 & 0.391 \\
& & $(0.322)$ & $(0.558)$ & $(0.361)$ & $(0.269)$ \\
& & & & & \\
& BSIV & 0.195 & 0.187 & 0.350 & 0.359 \\
& MFIV & 0.196 & 0.196 & 0.375 & 0.355 \\
& & $(0.818)$ & $(0.334)$ & $(0.501)$ & $(1.106)$ \\
& EBIV & 0.197 & 0.193 & 0.384 & 0.387 \\
& & $(0.668)$ & $(0.541)$ & $(0.308)$ & $(0.310)$ \\
\hline
\end{tabular}

Note: This table reports the estimated implied volatility calculated from 14 or 6 options with one-month maturity by the Black-Scholes formula (BSIV), the model-free method (MFIV) and the maximum entropy method (EBIV) under different risk neutral distributions. The first row provides the true volatilities of the underlying risk neutral distribution. The second row presents number of options used in calculating implied volatilities. The moneynesses of the 14 options range from 0.85 to 1.15 and the moneynesses of the 6 options range from 0.95 to 1.05 , both with equal interval 0.025. The degree of freedom of the Student-t and the two skewed Student-t distributions is 5. For the two skewed Student-t distributions, the skewness parameters are -0.3 and -0.7 , for "Skew1" and "Skew2" respectively. The estimation improvements of the MFIV and the EBIV compared to the BSIV are presented in parenthesis, which is defined as $\frac{\mid X X I V-\text { TrueVolatility } \mid}{\mid B S I V-\text { TrueVolatility } \mid}$, where XXIV is either EBIV or MFIV. 
Table 3: Coverage rates of the confidence intervals around the EBIV

\begin{tabular}{llllll}
\hline volatility & & Normal & Student t & Skewt1 & Skewt2 \\
\hline 0.2 & $95 \%$ & $91.21 \%$ & $91.40 \%$ & $92.30 \%$ & $93.10 \%$ \\
& $90 \%$ & $85.00 \%$ & $85.50 \%$ & $86.60 \%$ & $92.30 \%$ \\
0.4 & $95 \%$ & $92.39 \%$ & $91.60 \%$ & $93.40 \%$ & $92.50 \%$ \\
& $90 \%$ & $88.78 \%$ & $86.70 \%$ & $87.60 \%$ & $84.50 \%$ \\
\hline
\end{tabular}

Note: This table reports the coverage rates of confidence intervals under different risk neutral distributions. The upper panel is for $\sigma=0.2$ under $95 \%$ and $90 \%$ confidence levels and the lower panel is for $\sigma=0.4$. The degree of freedom of the Student-t and the two skewed Student- $t$ distributions is 5. For the two skewed Student- $t$ distributions, the skewness parameters are -0.3 and -0.7 , for "Skew1" and "Skew2" respectively. The details on how to construct the confidence interval of the EBIV are given in Section 2.2. 
Table 4: Comparison across the three methods: 3-month maturity

\begin{tabular}{|c|c|c|c|c|c|c|c|}
\hline & Volatility & $\begin{array}{r}0.2 \\
14\end{array}$ & $\begin{array}{r}0.2 \\
6\end{array}$ & $\begin{array}{r}0.2 \\
3+7\end{array}$ & $\begin{array}{r}0.4 \\
14\end{array}$ & $\begin{array}{r}0.4 \\
6\end{array}$ & $\begin{array}{r}0.4 \\
3+7\end{array}$ \\
\hline \multirow{3}{*}{ Normal } & BSIV & 0.200 & 0.200 & 0.200 & 0.400 & 0.400 & 0.400 \\
\hline & MFIV & 0.200 & 0.200 & 0.200 & 0.400 & 0.400 & 0.400 \\
\hline & EBIV & 0.201 & 0.202 & 0.200 & 0.404 & 0.417 & 0.405 \\
\hline \multirow[t]{5}{*}{ Student-t } & BSIV & 0.195 & 0.192 & 0.200 & 0.384 & 0.376 & 0.379 \\
\hline & MFIV & 0.194 & 0.193 & 0.204 & 0.386 & 0.376 & 0.382 \\
\hline & & (1.086) & (0.908) & $(8.784)$ & $(0.862)$ & $(0.995)$ & $(0.854)$ \\
\hline & EBIV & 0.197 & 0.196 & 0.197 & 0.395 & 0.397 & 0.393 \\
\hline & & $(0.515)$ & $(0.453)$ & $(6.329)$ & $(0.328)$ & $(0.131)$ & $(0.343)$ \\
\hline \multirow[t]{5}{*}{ Skewt1 } & BSIV & 0.192 & 0.188 & 0.199 & 0.368 & 0.361 & 0.370 \\
\hline & MFIV & 0.194 & 0.191 & 0.208 & 0.379 & 0.363 & 0.378 \\
\hline & & $(0.746)$ & $(0.762)$ & $(10.454)$ & $(0.664)$ & $(0.941)$ & $(0.714)$ \\
\hline & EBIV & 0.197 & 0.196 & 0.197 & 0.391 & 0.393 & 0.391 \\
\hline & & $(0.343)$ & $(0.359)$ & $(3.486)$ & $(0.274)$ & $(0.185)$ & $(0.302)$ \\
\hline \multirow[t]{5}{*}{ Skewt2 } & BSIV & 0.184 & 0.177 & 0.190 & 0.341 & 0.341 & 0.354 \\
\hline & MFIV & 0.191 & 0.186 & 0.208 & 0.367 & 0.346 & 0.369 \\
\hline & & $(0.534)$ & $(0.581)$ & $(0.763)$ & $(0.563)$ & $(0.902)$ & $(0.659)$ \\
\hline & EBIV & 0.195 & 0.193 & 0.195 & 0.384 & 0.384 & 0.385 \\
\hline & & $(0.290)$ & $(0.310)$ & $(0.471)$ & $(0.281)$ & $(0.262)$ & (0.329) \\
\hline
\end{tabular}

Note: This table reports the estimated implied volatility calculated from 14 or 6 options with three-month maturity by the Black-Scholes formula (BSIV), the model-free method (MFIV) and the maximum entropy method (EBIV) under different risk neutral distributions. The first row provides the true volatilities of the underlying risk neutral distribution. The second row presents number of options used in calculating implied volatilities. The moneynesses of the 14 options range from 0.85 to 1.15 and the moneynesses of the 6 options range from 0.95 to 1.05 , both with equal interval 0.025. The degree of freedom of the Student-t and the two skewed Student-t distributions is 5. For the two skewed Student-t distributions, the skewness parameters are -0.3 and -0.7 , for "Skew1" and "Skew2" respectively. The estimation improvements of the MFIV and the EBIV compared to the BSIV are presented in parenthesis, which is defined as $\frac{\mid X X I V-\text { TrueVolatility } \mid}{\mid B S I V-\text { TrueV olatility } \mid}$, where XXIV is either EBIV or MFIV. 
Table 5: Implied volatilities estimated using the three methods: 1-year maturity

\begin{tabular}{|c|c|c|c|c|c|c|c|}
\hline & Volatility & 0.2 & 0.2 & 0.2 & 0.4 & 0.4 & $\begin{array}{r}0.4 \\
\end{array}$ \\
\hline & Option No. & 14 & 6 & $3+7$ & 14 & 6 & $3+7$ \\
\hline \multirow[t]{3}{*}{ Normal } & BSIV & 0.200 & 0.200 & 0.200 & 0.400 & 0.400 & 0.400 \\
\hline & MFIV & 0.200 & 0.200 & 0.200 & 0.400 & 0.400 & 0.400 \\
\hline & EBIV & 0.204 & 0.202 & 0.200 & 0.407 & 0.423 & 0.407 \\
\hline \multirow[t]{5}{*}{ Student-t } & BSIV & 0.188 & 0.192 & 0.198 & 0.392 & 0.386 & 0.388 \\
\hline & MFIV & 0.190 & 0.192 & 0.199 & 0.390 & 0.381 & 0.387 \\
\hline & & $(0.850)$ & $(0.933)$ & (0.598) & $(1.363)$ & (1.328) & (1.037) \\
\hline & EBIV & 0.197 & 0.197 & 0.197 & 0.401 & 0.406 & 0.399 \\
\hline & & $(0.256)$ & $(0.402)$ & (1.181) & $(0.101)$ & $(0.452)$ & $(0.072)$ \\
\hline \multirow[t]{5}{*}{ Skewt1 } & BSIV & 0.191 & 0.193 & 0.201 & 0.366 & 0.363 & 0.368 \\
\hline & MFIV & 0.193 & 0.193 & 0.203 & 0.382 & 0.368 & 0.382 \\
\hline & & $(0.844)$ & $(0.977)$ & $(3.253)$ & $(0.536)$ & $(0.867)$ & $(0.572)$ \\
\hline & EBIV & 0.200 & 0.199 & 0.201 & 0.399 & 0.404 & 0.398 \\
\hline & & $(0.040)$ & $(0.086)$ & (0.651) & $(0.036)$ & $(0.112)$ & $(0.049)$ \\
\hline \multirow[t]{5}{*}{ Skewt2 } & BSIV & 0.188 & 0.183 & 0.193 & 0.331 & 0.334 & 0.342 \\
\hline & MFIV & 0.190 & 0.187 & 0.200 & 0.366 & 0.346 & 0.369 \\
\hline & & $(0.840)$ & $(0.810)$ & $(0.054)$ & $(0.486)$ & $(0.812)$ & $(0.546)$ \\
\hline & EBIV & 0.193 & 0.196 & 0.198 & 0.389 & 0.393 & 0.390 \\
\hline & & $(0.542)$ & $(0.253)$ & $(0.316)$ & $(0.163)$ & $(0.102)$ & $(0.175)$ \\
\hline
\end{tabular}

Note: This table reports the estimated implied volatility calculated from 14 or 6 options with one-year maturity by the Black-Scholes formula (BSIV), the model-free method (MFIV) and the maximum entropy method (EBIV) under different risk neutral distributions. The first row provides the true volatilities of the underlying risk neutral distribution. The second row presents number of options used in calculating implied volatilities. The moneynesses of the 14 options range from 0.85 to 1.15 and the moneynesses of the 6 options range from 0.95 to 1.05 , both with equal interval 0.025. The degree of freedom of the Student- $t$ and the two skewed Student-t distributions is 5 . For the two skewed Student-t distributions, the skewness parameters are -0.3 and -0.7 , for "Skew1" and "Skew2" respectively. The estimation improvements of the MFIV and the EBIV compared to the BSIV are presented in parenthesis, which is defined as $\frac{\mid X X I V-\text { TrueVolatility } \mid}{\mid B S I V-\text { TrueVolatility } \mid}$, where XXIV is either EBIV or MFIV. 
Table 6: Implied moments for the stochastic volatility and jump (SVJ) model

\begin{tabular}{rrrr|rrrr}
\hline & \multicolumn{3}{c}{ Parameter set I } & \multicolumn{4}{c}{ Parameter set II } \\
\hline & volatility & skewness & kurtosis & & volatility & skewness & kurtosis \\
true value & 0.203 & -0.401 & 3.179 & true moments & 0.453 & -0.554 & 3.53 \\
BSIV & 0.2 & - & - & BSIV & 0.445 & - & - \\
MFIV & 0.205 & -0.274 & 2.924 & MFIV & 0.427 & -0.059 & 1.76 \\
EBIV & 0.203 & -0.404 & 3.207 & EBIV & 0.454 & -0.543 & 3.435 \\
\hline
\end{tabular}

Note: This table provides a comparison between the three methods, the Black-Scholes formula (BSIV), the model-free (MFIV) method and the entropy-based (EBIV) method, when the option prices are simulated from the stochastic volatility and jump (SVJ) model (for details, see Section 3.4). For the two sets of parameters given in Section 3.4, the true moments of the underlying distribution are presented in the first row and the implied moments calculated from the three methods are presented in the second to fourth rows. The true moments of the risk neutral distributions are determined by pre-simulating 100,000 monthly returns from the SVJ model. Option prices and the true moments are calculated based on the simulated monthly returns. There are 14 options in total, with one-month maturity. The moneynesses of the options range from 0.85 to 1.15 with equal interval 0.025 . 
Table 7: Descriptive statistics of the S\&P500 index options with 1 month expiration

(a) Panel A: Call options

\begin{tabular}{rrrrrrrr}
\hline$K_{c} / S$ & 1 & 1.025 & 1.05 & 1.075 & 1.1 & 1.125 & 1.15 \\
\hline Mean & 24.62 & 11.38 & 4.78 & 2.23 & 1.28 & 0.93 & 0.77 \\
Variance & 80.02 & 65.66 & 33.11 & 15.39 & 7.36 & 4.35 & 2.28 \\
Skewness & 1.11 & 1.66 & 3.15 & 5.26 & 7.17 & 8.66 & 9.73 \\
Maximum & 7.69 & 1.28 & 0.40 & 0.40 & 0.40 & 0.40 & 0.40 \\
Minimum & 64.85 & 53.25 & 45.20 & 36.80 & 29.30 & 24.50 & 19.30 \\
obs. & 222 & 222 & 222 & 196 & 123 & 57 & 33 \\
\hline
\end{tabular}

(b) Panel B: Put options

\begin{tabular}{rrrrrrrr}
\hline$K_{p} / S$ & 0.85 & 0.875 & 0.9 & 0.925 & 0.95 & 0.975 & 1 \\
\hline Mean & 1.96 & 2.69 & 3.91 & 5.78 & 8.92 & 14.38 & 24.10 \\
Variance & 9.06 & 13.37 & 20.66 & 30.97 & 47.59 & 66.61 & 80.86 \\
Skewness & 4.57 & 4.12 & 3.41 & 2.83 & 2.25 & 1.66 & 0.99 \\
Maximum & 0.40 & 0.40 & 0.40 & 0.53 & 1.33 & 3.65 & 8.25 \\
Minimum & 25.20 & 30.05 & 34.45 & 40.35 & 47.35 & 55.25 & 62.65 \\
obs. & 222 & 190 & 216 & 220 & 221 & 221 & 222 \\
\hline
\end{tabular}

Note: This table reports descriptive statistics of the S\&P500 call and put options with 1 month expiration from January 1996 to August 2014. The options are selected by the procedure illustrated in Section 4.1. The first row in Panel 7a (Panel 7b) shows moneynesses of the out-of-the-money call (put) options, where $K_{c}\left(K_{p}\right)$ are exercise prices of the call (put) options, $S$ is the current price of the S\&P500 index. The last row labelled "obs" shows the number of observations in each moneyness category. 
Table 8: Descriptive statistics of different measures of volatility

\begin{tabular}{rrrrrrrr}
\hline & Mean & Median & Std. Dev. & Skewness & Kurtosis & Maximum & Minimum \\
\hline RV & 0.172 & 0.145 & 0.101 & 2.739 & 14.158 & 0.784 & 0.055 \\
VIX & 0.217 & 0.200 & 0.093 & 2.460 & 13.122 & 0.809 & 0.102 \\
BSIV & 0.219 & 0.204 & 0.078 & 2.994 & 17.341 & 0.765 & 0.130 \\
MFIV & 0.209 & 0.195 & 0.085 & 2.460 & 13.349 & 0.758 & 0.103 \\
EBIV & 0.210 & 0.194 & 0.089 & 2.257 & 11.343 & 0.745 & 0.097 \\
\hline
\end{tabular}

Note: This table reports the descriptive statistics for the volatility measures: RV, VIX, BSIV, MFIV and EBIV. RV is the realized volatility of the preceding 30 days defined in 12 . VIX is the volatility index provided by CBOE. BSIV is the average Black-Scholes implied volatility calculated from all available option prices. MFIV is calculated based on Appendix 6.1. The details of calculating EBIV are given in Section 2.1 and 4.1. Statistics are reported for the full sample from January 1996 to August 2014. The volatility measures are annualized and given in decimal form.

Table 9: Correlation matrix of different measures of volatilities

\begin{tabular}{rrrrrr}
\hline & RV & VIX & BSIV & MFIV & EBIV \\
\hline RV & 1.000 & 0.734 & 0.735 & 0.735 & 0.735 \\
VIX & 0.734 & 1.000 & 0.994 & 0.998 & 0.998 \\
BSIV & 0.735 & 0.994 & 1.000 & 0.996 & 0.993 \\
MFIV & 0.735 & 0.998 & 0.996 & 1.000 & 0.998 \\
EBIV & 0.735 & 0.998 & 0.993 & 0.998 & 1.000 \\
\hline
\end{tabular}

Note: This table reports the correlation coefficients across the volatilities measures: RV, VIX, BSIV, MFIV and EBIV. RV is the realized volatility of the preceding 30 days defined in 12 . VIX is the volatility index provided by CBOE. BSIV is the average Black-Scholes implied volatility calculated from all available option prices. MFIV is calculated based on Appendix 6.1. The details of calculating EBIV are given in Section 2.1 and 4.1. The volatility measures are annualized and given in decimal form. The sample period is from January 1996 to August 2014. 
Table 10: Predict the realized volatility: volatility regressions

\begin{tabular}{|c|c|c|c|c|}
\hline \multicolumn{5}{|c|}{ Panel A: Univariate regressions of the 5 volatility measures } \\
\hline & $\alpha$ & $\beta_{1}$ & $\beta_{2}$ & $\operatorname{adj} . R^{2}$ \\
\hline \multirow[t]{2}{*}{ RV } & $0.050^{* * *}$ & $0.680^{* * *}$ & - & 0.486 \\
\hline & $(3.681)$ & $(7.544)$ & - & \\
\hline \multirow[t]{2}{*}{ BSIV } & -0.024 & $0.917^{* * *}$ & - & 0.554 \\
\hline & $-(1.428)$ & $(9.982)$ & - & \\
\hline \multirow[t]{2}{*}{ VIX } & -0.003 & $0.805^{* * *}$ & - & 0.554 \\
\hline & $-(0.221)$ & (9.929) & - & \\
\hline \multirow[t]{2}{*}{ MFIV } & -0.006 & $0.823^{* * *}$ & - & 0.559 \\
\hline & $-(0.419)$ & $(9.879)$ & - & \\
\hline \multirow[t]{2}{*}{ EBIV } & 0.005 & $0.787^{* * *}$ & - & 0.564 \\
\hline & $(0.397)$ & $(10.401)$ & - & \\
\hline \multicolumn{5}{|c|}{ Panel B: Bivariate regressions (volatility measures and uncorrelated EBIV residuals) } \\
\hline & $\alpha$ & $\beta_{1}$ & $\beta_{2}$ & $\operatorname{adj} . R^{2}$ \\
\hline \multirow[t]{2}{*}{$\mathrm{RV}+\epsilon_{E B I V, R V}$} & $0.050 * * *$ & $0.679^{* * *}$ & $0.655^{* * *}$ & 0.571 \\
\hline & $(6.285)$ & $(11.873)$ & $(5.944)$ & \\
\hline \multirow[t]{2}{*}{$\mathrm{BSIV}+\epsilon_{E B I V, B S I V}$} & $-0.029^{* * *}$ & $0.926^{* * *}$ & $1.675^{*}$ & 0.570 \\
\hline & $-(2.631)$ & $(15.179)$ & $(1.570)$ & \\
\hline \multirow[t]{2}{*}{$\mathrm{VIX}+\epsilon_{E B I V, V I X}$} & -0.003 & $0.803^{* * *}$ & $1.424^{* *}$ & 0.570 \\
\hline & $-(0.292)$ & $(16.099)$ & $(2.332)$ & \\
\hline \multirow[t]{2}{*}{$\mathrm{MFIV}+\epsilon_{E B I V, M F I V}$} & -0.006 & $0.822^{* * *}$ & $1.082^{*}$ & 0.567 \\
\hline & $-(0.596)$ & $(15.026)$ & $(1.722)$ & \\
\hline \\
\hline \multicolumn{5}{|c|}{ 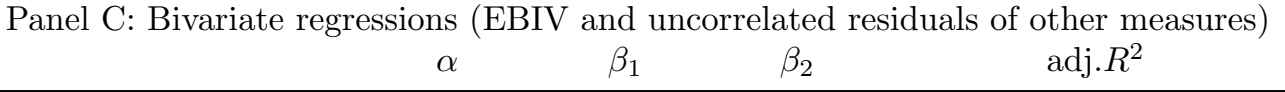 } \\
\hline \multirow[t]{2}{*}{$\mathrm{EBIV}+\epsilon_{R V, E B I V}$} & 0.006 & $0.785^{* * *}$ & 0.136 & 0.571 \\
\hline & $(0.641)$ & $(14.952)$ & $(1.124)$ & \\
\hline \multirow[t]{2}{*}{$\mathrm{EBIV}+\epsilon_{B S I V, E B I V}$} & 0.006 & $0.785^{* * *}$ & -0.941 & 0.570 \\
\hline & $(0.637)$ & $(14.760)$ & $-(0.804)$ & \\
\hline \multirow[t]{2}{*}{$\mathrm{EBIV}+\epsilon_{V I X, E B I V}$} & 0.006 & $0.785^{* * *}$ & -0.662 & 0.570 \\
\hline & $(0.643)$ & $(15.850)$ & $-(1.074)$ & \\
\hline \multirow[t]{2}{*}{$\mathrm{EBIV}+\epsilon_{M F I V, E B I V}$} & 0.006 & $0.785^{* * *}$ & -0.313 & 0.567 \\
\hline & $(0.629)$ & $(14.791)$ & $-(0.486)$ & \\
\hline
\end{tabular}

Note: This table reports the results for predicting future realized volatility using different measures of volatility. All regressions are based on monthly non-overlapping observations. The dependent variable is the realized volatility in the next month defined in equation (12). Robust t-statistics are reported in parentheses taking into account the heteroscedastic and autocorrelated error structure. 
Table 11: Out-of-sample Diebold-Mariano-West test

\begin{tabular}{|c|c|c|c|c|c|c|c|c|c|}
\hline \multicolumn{5}{|c|}{ Panel A: Full sample } & \multicolumn{5}{|c|}{ Panel B: Low volatility period } \\
\hline & BSIV & VIX & MFIV & EBIV & & BSIV & VIX & MFIV & EBIV \\
\hline $\mathrm{RV}$ & $\begin{array}{c}0.928 \\
(0.754)\end{array}$ & $\begin{array}{c}0.922 \\
(0.858)\end{array}$ & $\begin{array}{c}0.915 \\
(0.963)\end{array}$ & $\begin{array}{c}0.890 \\
(1.229)\end{array}$ & RV & $\begin{array}{c}0.342^{* * *} \\
(5.284)\end{array}$ & $\begin{array}{c}0.317^{* * *} \\
(5.589)\end{array}$ & $\begin{array}{c}0.316^{* * *} \\
(5.676)\end{array}$ & $\begin{array}{c}0.317^{* * *} \\
(5.845)\end{array}$ \\
\hline BSIV & & $\begin{array}{c}0.993 \\
(0.263)\end{array}$ & $\begin{array}{c}0.986 \\
(0.485)\end{array}$ & $\begin{array}{c}0.959 \\
(1.154)\end{array}$ & BSIV & & $\begin{array}{c}0.926^{* * *} \\
(2.996)\end{array}$ & $\begin{array}{c}0.925^{* *} \\
(2.187)\end{array}$ & $\begin{array}{c}0.927 \\
(1.573)\end{array}$ \\
\hline VIX & & & $\begin{array}{c}0.993 \\
(0.924)\end{array}$ & $\begin{array}{l}0.966^{*} \\
(1.678)\end{array}$ & VIX & & & $\begin{array}{c}0.999 \\
(0.032)\end{array}$ & $\begin{array}{c}1.001 \\
(-0.040)\end{array}$ \\
\hline MFIV & & & & $\begin{array}{l}0.973^{*} \\
(1.779)\end{array}$ & MFIV & & & & $\begin{array}{c}1.002 \\
(-0.051)\end{array}$ \\
\hline \multicolumn{5}{|c|}{ Panel C: Medium volatility period } & \multicolumn{5}{|c|}{ Panel D: High volatility period } \\
\hline & BSIV & VIX & MFIV & EBIV & & BSIV & VIX & MFIV & EBIV \\
\hline RV & $\begin{array}{c}0.986 \\
(0.163)\end{array}$ & $\begin{array}{c}0.956 \\
(0.539)\end{array}$ & $\begin{array}{c}0.961 \\
(0.505)\end{array}$ & $\begin{array}{c}0.959 \\
(0.503)\end{array}$ & $\mathrm{RV}$ & $\begin{array}{c}0.975 \\
(0.165)\end{array}$ & $\begin{array}{c}0.986 \\
(0.093)\end{array}$ & $\begin{array}{c}0.971 \\
(0.196)\end{array}$ & $\begin{array}{c}0.928 \\
(0.474)\end{array}$ \\
\hline BSIV & & $\begin{array}{c}0.970^{* * * *} \\
(2.269)\end{array}$ & $\begin{array}{c}0.975 \\
(1.486)\end{array}$ & $\begin{array}{c}0.973^{* *} \\
(1.919)\end{array}$ & BSIV & & $\begin{array}{c}1.011 \\
(-0.292)\end{array}$ & $\begin{array}{c}0.996 \\
(0.082)\end{array}$ & $\begin{array}{c}0.952 \\
(0.899)\end{array}$ \\
\hline VIX & & & $\begin{array}{c}1.005 \\
(-0.456)\end{array}$ & $\begin{array}{c}1.003 \\
(-0.364)\end{array}$ & VIX & & & $\begin{array}{c}0.985 \\
(1.170)\end{array}$ & $\begin{array}{c}0.942^{* *} \\
(2.101)\end{array}$ \\
\hline MFIV & & & & $\begin{array}{c}0.998 \\
(0.219)\end{array}$ & MFIV & & & & $\begin{array}{c}0.956^{* *} \\
(2.006)\end{array}$ \\
\hline
\end{tabular}

Note: This table reports the MFSE ratio $\left(\frac{M F S E_{j}}{M F S E_{i}}\right)$ and DMW statistics in the parenthesis, where $i$ represent the implied volatility measures on the first column and $j$ represent those on the first row. Diebold and Mariano (1995) and West (1996) (DMW) statistics are computed to test the null of equal predictive ability) $\left(M F S E_{j}=M F S E_{i}\right)$ against the alternative that the competing model has a lower MSFE than the baseline model $\left(M F S E_{j}>M F S E_{i}\right)$.

Table 12: Diebold-Mariano-West test: nested models

\begin{tabular}{lllll}
\hline & RV & BSIV & VIX & MFIV \\
\hline $\operatorname{MFSE}\left(\{X\},\left\{X, \epsilon_{E B I V, X}\right\}\right)$ & $\begin{array}{l}0.894^{* * *} \\
(3.956)\end{array}$ & $\begin{array}{l}0.983^{* * *} \\
(2.231)\end{array}$ & $\begin{array}{l}0.986^{* * *} \\
(2.061)\end{array}$ & $\begin{array}{l}1.052 \\
(-0.383)\end{array}$ \\
$\operatorname{MFSE}\left(\{E B I V\},\left\{E B I V, \epsilon_{X, E B I V}\right\}\right)$ & 1.005 & 1.024 & 1.021 & 1.082 \\
& $(0.506)$ & $(1.196)$ & $(-0.382)$ & $(-0.335)$ \\
\hline
\end{tabular}

Note: This table reports the MFSE ratio and the DMW statistics for nested models. The MFSE ratio is defined as $\operatorname{MFSE}\left(X_{i}, X_{j}\right)=\frac{M F S E\left(X_{j}\right)}{M F S E\left(X_{i}\right)}$ and the DMW statistics is calculated after adjusting for nested models suggested in Clark and West (2007). 
Table 13: Predict the realized volatility: log volatility regressions

\begin{tabular}{|c|c|c|c|c|}
\hline \multicolumn{5}{|c|}{ Panel A: Univariate regressions of the 5 volatility measures } \\
\hline $\mathrm{RV}$ & $\begin{array}{c}-0.617^{* * *} \\
(-5.237)\end{array}$ & $\begin{array}{r}0.690^{* * *} \\
(11.817)\end{array}$ & - & 0.461 \\
\hline BSIV & $\begin{array}{l}-0.038 \\
(-0.379)\end{array}$ & $\begin{array}{l}1.169^{* * *} \\
(19.924)\end{array}$ & - & 0.587 \\
\hline VIX & $\begin{array}{c}-0.293^{* * *} \\
(-2.849)\end{array}$ & $\begin{array}{r}0.996^{* * *} \\
(17.583)\end{array}$ & - & 0.589 \\
\hline MFIV & $\begin{array}{c}-0.271^{* * *} \\
(-2.652)\end{array}$ & $\begin{array}{l}1.008^{* * *} \\
(17.860)\end{array}$ & - & 0.593 \\
\hline EBIV & $\begin{array}{c}-0.305^{* * *} \\
(-3.146)\end{array}$ & $\begin{array}{c}0.969^{* * *} \\
(18.550)\end{array}$ & - & 0.593 \\
\hline \multicolumn{5}{|c|}{ 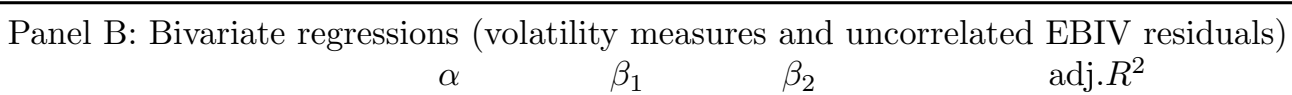 } \\
\hline $\mathrm{RV}+\epsilon_{E B I V, R V}$ & $\begin{array}{c}-0.617^{* * *} \\
(-6.286)\end{array}$ & $\begin{array}{c}0.690^{* * *} \\
(14.800)\end{array}$ & $\begin{array}{c}0.924^{* * *} \\
(9.510)\end{array}$ & 0.596 \\
\hline $\mathrm{BSIV}+\epsilon_{E B I V, B S I V}$ & $\begin{array}{c}-0.038 \\
(-0.344)\end{array}$ & $\begin{array}{l}1.169^{* * *} \\
(18.404)\end{array}$ & $\begin{array}{l}1.131^{*} \\
(1.744)\end{array}$ & 0.596 \\
\hline $\mathrm{VIX}+\epsilon_{E B I V, V I X}$ & $\begin{array}{c}-0.293 \\
(-3.106)\end{array}$ & $\begin{array}{c}0.996^{* * *} \\
(19.116)\end{array}$ & $\begin{array}{l}1.366 \\
(1.544)\end{array}$ & 0.596 \\
\hline $\mathrm{MFIV}+\epsilon_{E B I V, M F I V}$ & $\begin{array}{c}-0.271 \\
(-2.708)\end{array}$ & $\begin{array}{c}1.008^{* * *} \\
(18.262)\end{array}$ & $\begin{array}{c}0.461 \\
(0.345)\end{array}$ & 0.596 \\
\hline \multicolumn{5}{|c|}{ Panel C: Bivariate regressions (EBIV and uncorrelated residuals of other measures) } \\
\hline $\mathrm{EBIV}+\epsilon_{R V, E B I V}$ & $\begin{array}{c}-0.305^{* * *} \\
(-3.209)\end{array}$ & $\begin{array}{c}0.969^{* * *} \\
(18.874)\end{array}$ & $\begin{array}{c}0.042 \\
(0.471)\end{array}$ & 0.596 \\
\hline $\mathrm{EBIV}+\epsilon_{B S I V, E B I V}$ & $\begin{array}{c}-0.305^{* * *} \\
(-3.085)\end{array}$ & $\begin{array}{c}0.969^{* * * *} \\
(18.212)\end{array}$ & $\begin{array}{c}-0.197 \\
(-0.255)\end{array}$ & 0.596 \\
\hline $\mathrm{EBIV}+\epsilon_{V I X, E B I V}$ & $\begin{array}{c}-0.305^{* * *} \\
(-3.220)\end{array}$ & $\begin{array}{c}0.969^{* * *} \\
(18.975)\end{array}$ & $\begin{array}{c}-0.410 \\
(-0.454)\end{array}$ & 0.596 \\
\hline $\mathrm{EBIV}+\epsilon_{M F I V, E B I V}$ & $\begin{array}{c}-0.305^{* * *} \\
(-3.078)\end{array}$ & $\begin{array}{c}0.969^{* * *} \\
(18.133)\end{array}$ & $\begin{array}{c}0.529 \\
(0.382)\end{array}$ & 0.596 \\
\hline
\end{tabular}

Note: This table reports the results for predicting future log realized volatility using different measures of log volatility. All regressions are based on monthly non-overlapping observations. The dependent variable is the realized volatility in the next month defined in equation (12). Robust t-statistics are reported in parentheses taking into account the heteroscedastic and autocorrelated error structure. 
Table 14: Out-of-sample Diebold-Mariano-West test: log volatility regressions

\begin{tabular}{|c|c|c|c|c|c|c|c|c|c|}
\hline \multicolumn{5}{|c|}{ Panel A: Full sample } & \multicolumn{5}{|c|}{ Panel B: Low volatility period } \\
\hline & BSIV & VIX & MFIV & EBIV & & BSIV & VIX & MFIV & EBIV \\
\hline RV & $\begin{array}{c}0.772^{* * *} \\
(3.330)\end{array}$ & $\begin{array}{c}0.762^{* * *} \\
(3.387)\end{array}$ & $\begin{array}{c}0.760^{* * *} \\
(3.468)\end{array}$ & $\begin{array}{c}0.751^{* * *} \\
(3.601)\end{array}$ & RV & $\begin{array}{c}0.513^{* * *} \\
(4.435)\end{array}$ & $\begin{array}{c}0.491^{* * *} \\
(4.592)\end{array}$ & $\begin{array}{c}0.493^{* * *} \\
(4.559)\end{array}$ & $\begin{array}{c}0.485^{* * *} \\
(4.727)\end{array}$ \\
\hline BSIV & & $\begin{array}{c}0.986 \\
(0.639)\end{array}$ & $\begin{array}{c}0.984 \\
(0.657)\end{array}$ & $\begin{array}{c}0.973 \\
(1.060)\end{array}$ & BSIV & 0.000 & $\begin{array}{c}0.957 \\
(1.605)\end{array}$ & $\begin{array}{c}0.962 \\
(1.005)\end{array}$ & $\begin{array}{c}0.945 \\
(1.401)\end{array}$ \\
\hline VIX & & & $\begin{array}{c}0.998 \\
(0.261)\end{array}$ & $\begin{array}{c}0.986 \\
(1.242)\end{array}$ & VIX & & & $\begin{array}{c}1.005 \\
-(0.216)\end{array}$ & $\begin{array}{c}0.987 \\
(0.528)\end{array}$ \\
\hline MFIV & & & & $\begin{array}{c}0.988 \\
(1.394)\end{array}$ & MFIV & & & & $\begin{array}{c}0.982 \\
(1.039)\end{array}$ \\
\hline \multicolumn{5}{|c|}{ Panel C: Medium volatility period } & \multicolumn{5}{|c|}{ Panel D: High volatility period } \\
\hline & BSIV & VIX & MFIV & EBIV & & BSIV & VIX & MFIV & EBIV \\
\hline RV & $\begin{array}{c}0.863 \\
(1.343)\end{array}$ & $\begin{array}{c}0.830^{*} \\
(1.695)\end{array}$ & $\begin{array}{c}0.833^{*} \\
(1.779)\end{array}$ & $\begin{array}{c}0.830^{*} \\
(1.745)\end{array}$ & $\mathrm{RV}$ & $\begin{array}{c}0.867 \\
(0.924)\end{array}$ & $\begin{array}{c}0.920 \\
(0.456)\end{array}$ & $\begin{array}{c}0.905 \\
(0.549)\end{array}$ & $\begin{array}{c}0.881 \\
(0.669)\end{array}$ \\
\hline BSIV & & $\begin{array}{c}0.961^{*} \\
(1.693)\end{array}$ & $\begin{array}{c}0.964 \\
(1.303)\end{array}$ & $\begin{array}{c}0.962 \\
(1.450)\end{array}$ & BSIV & & $\begin{array}{c}1.061 \\
(-1.058)\end{array}$ & $\begin{array}{c}1.043 \\
(-0.700)\end{array}$ & $\begin{array}{c}1.016 \\
(-0.224)\end{array}$ \\
\hline VIX & & & $\begin{array}{c}1.003 \\
(-0.227)\end{array}$ & $\begin{array}{c}1.001 \\
(-0.056)\end{array}$ & VIX & & & $\begin{array}{c}0.983 \\
(1.237)\end{array}$ & $\begin{array}{l}0.957^{*} \\
(1.771)\end{array}$ \\
\hline MFIV & & & & $\begin{array}{c}0.997 \\
(0.236)\end{array}$ & MFIV & & & & $\begin{array}{c}0.974 \\
(1.611)\end{array}$ \\
\hline
\end{tabular}

Note: This table reports the MFSE ratio $\left(\frac{M F S E_{j}}{M F S E_{i}}\right)$ and DMW statistics in the parenthesis, where $i$ represent the implied volatility measures on the first column and $j$ represent those on the first row. Diebold and Mariano (1995) and West (1996) (DMW) statistics are computed to test the null of equal predictive ability) $\left(M F S E_{j}=M F S E_{i}\right)$ against the alternative that the competing model has a lower MSFE than the baseline model $\left(M F S E_{j}>M F S E_{i}\right)$.

Table 15: Diebold-Mariano-West test: nested models

\begin{tabular}{lllll}
\hline & RV & BSIV & VIX & MFIV \\
\hline$M F S E\left(\{X\},\left\{X, \epsilon_{E B I V, X}\right\}\right)$ & $0.894^{* * *}$ & $0.983^{* *}$ & $0.986^{* *}$ & 1.052 \\
& $(3.956)$ & $(2.231)$ & $(2.061)$ & $(-0.383)$ \\
$M F S E\left(\{E B I V\},\left\{E B I V, \epsilon_{X, E B I V}\right\}\right)$ & 1.005 & 1.024 & 1.021 & 1.082 \\
& $(0.506)$ & $(1.196)$ & $(-0.382)$ & $(-0.335)$ \\
\hline
\end{tabular}

Note: This table reports the MFSE ratio and the DMW statistics for nested models. The MFSE ratio is defined as $\operatorname{MFSE}\left(X_{i}, X_{j}\right)=\frac{\operatorname{MFSE}\left(X_{j}\right)}{\operatorname{MFSE}\left(X_{i}\right)}$ and the DMW statistics is calculated after adjusting for nested models suggested in Clark and West (2007). 
Table 16: Predict the monthly returns using variance risk premium

\begin{tabular}{r|rrr|rrrr}
\hline & \multicolumn{3}{|c|}{ In-Sample Estimation } & \multicolumn{4}{|c}{ Out-of-sample MFSE } \\
& $\alpha$ & $\beta_{1}$ & adj. $R^{2}$ & All days & Low & Medium & High \\
\hline$V R P_{B S}$ & 0.002 & $0.353^{* * *}$ & 0.051 & $2.421 \mathrm{E}-03$ & $6.325 \mathrm{E}-04$ & $2.236 \mathrm{E}-03$ & $4.409 \mathrm{E}-03$ \\
& $(0.427)$ & $(3.916)$ & & & & & \\
$V R P_{M F}$ & 0.000 & $0.411^{* * *}$ & 0.065 & $2.385 \mathrm{E}-03$ & $6.620 \mathrm{E}-04$ & $2.243 \mathrm{E}-03$ & $4.242 \mathrm{E}-03$ \\
& $-(0.040)$ & $(4.430)$ & & & & & \\
$V R P_{E B}$ & 0.000 & $0.425^{* * *}$ & 0.071 & $2.389 \mathrm{E}-03$ & $6.589 \mathrm{E}-04$ & $2.242 \mathrm{E}-03$ & $4.259 \mathrm{E}-03$ \\
& $(0.046)$ & $(4.273)$ & & & & & \\
& & & & & & & \\
\hline \hline
\end{tabular}

Note: This table reports the results for predicting future monthly return using different variance risk premia. $V R P_{B S}$ is the variance risk premium calculated by the difference between $B S I V^{2}$ and realized variance in the last month $R V^{2}$, as defined in equation (12). $V R P_{M F}$ and $V R P_{E B}$ are variance risk premium calculated based on MFIV and EBIV in a similar way. The sample period extends from January 1996 to August 2014. In the panel In-sample Estimation, all regressions are based on monthly non-overlapping observations. The dependent variable is S\&P500 index return in the next month. Robust t-statistics are reported in parentheses taking into account the heteroscedastic and autocorrelated error structure. In the panel Out-of-sample MFSE, the forecasting are conducted based on a moving window of 100 observations preceding to the period to be forecasted. Besides the results for "All Days" in the forecasting period, the whole sample is further split into three sub-samples by sorting the BSIV in the month preceding to the forecasting period in ascending order, which results in three regimes: "Low", "Medium" and "High". The RMSEs are then calculated within each sub-sample. 
Figure 1: Comparing the estimated risk neutral distribution to the true distribution

(a) Standard normal

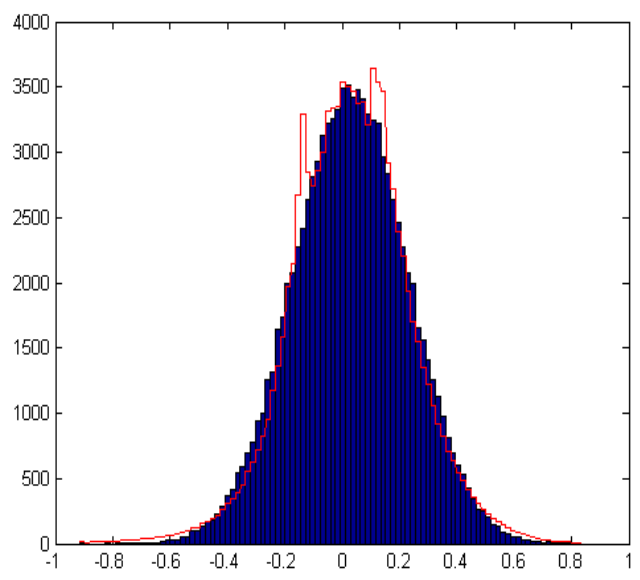

(c) Skewt1

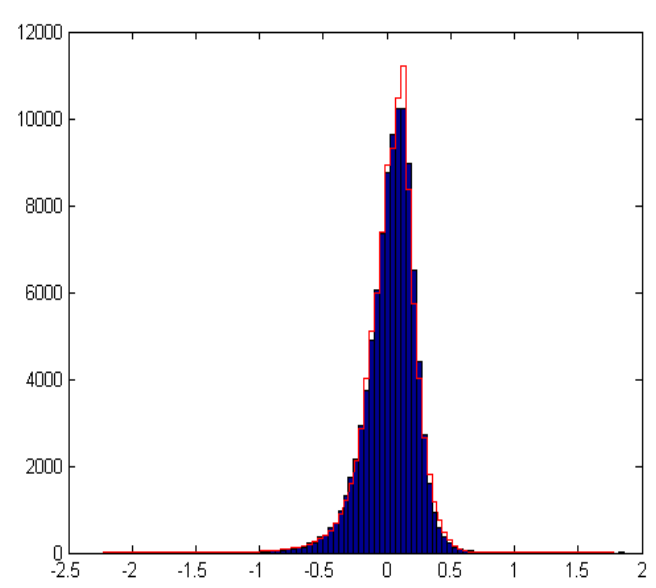

(b) Student-t

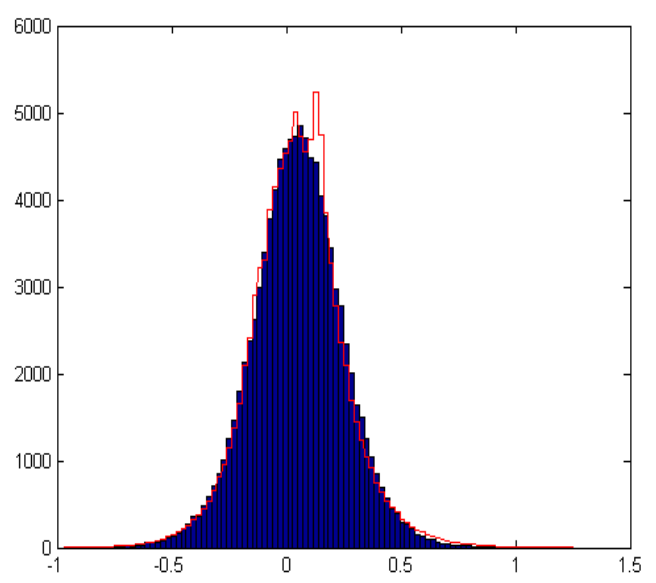

(d) Skewt2

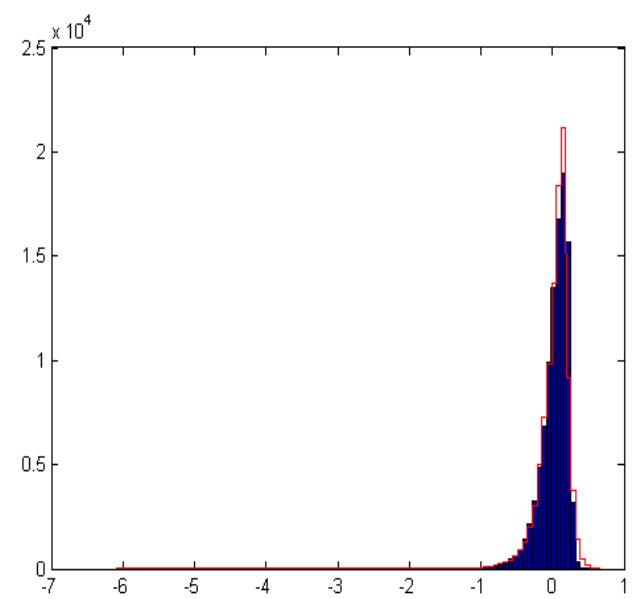

Note: This figure compares the estimated risk neutral distribution to the true distribution from the data generating process. The blue bars show the histogram of the simulated true distribution. The red lines show the estimated risk neutral densities using the Maximum Entropy method. The distributions are estimated from 14 options. Their moneynesses range from 0.85 to 1.15 with an equal interval 0.025 . The degree of freedom of the Student-t and the two skewed Student-t distributions is 5. For the two skewed Student- $t$ distributions, the skewness parameters are -0.3 and -0.7 , for "Skew1" and "Skew2" respectively. 
Figure 2: Comparison between the MFIV and the EBIV

(a) EBIV and MFIV

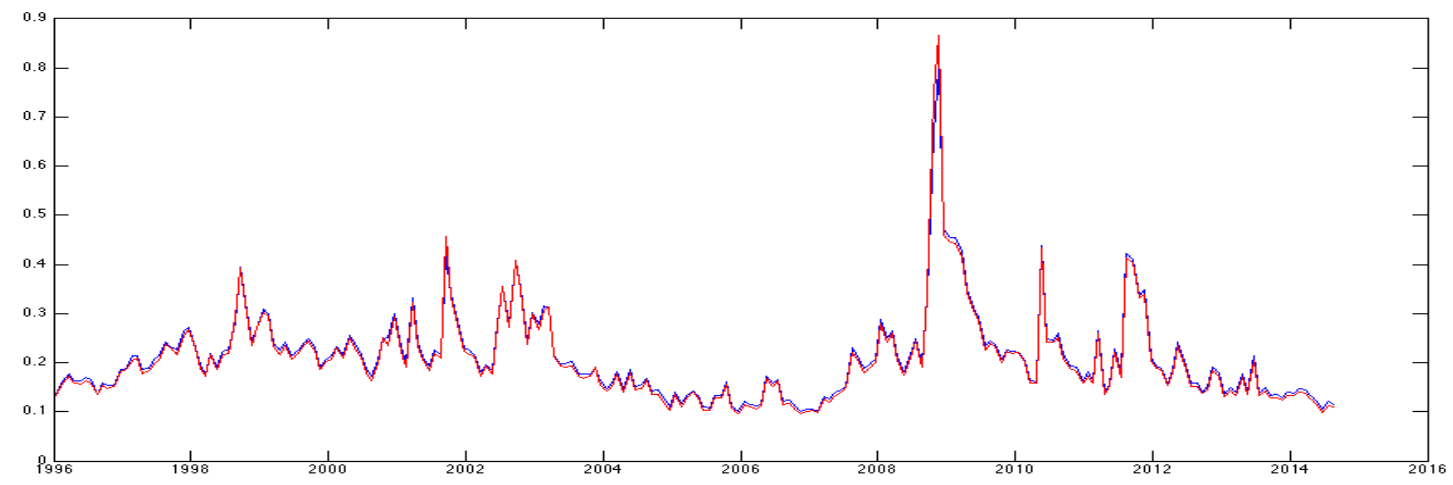

(b) Difference between EBIV and MFIV

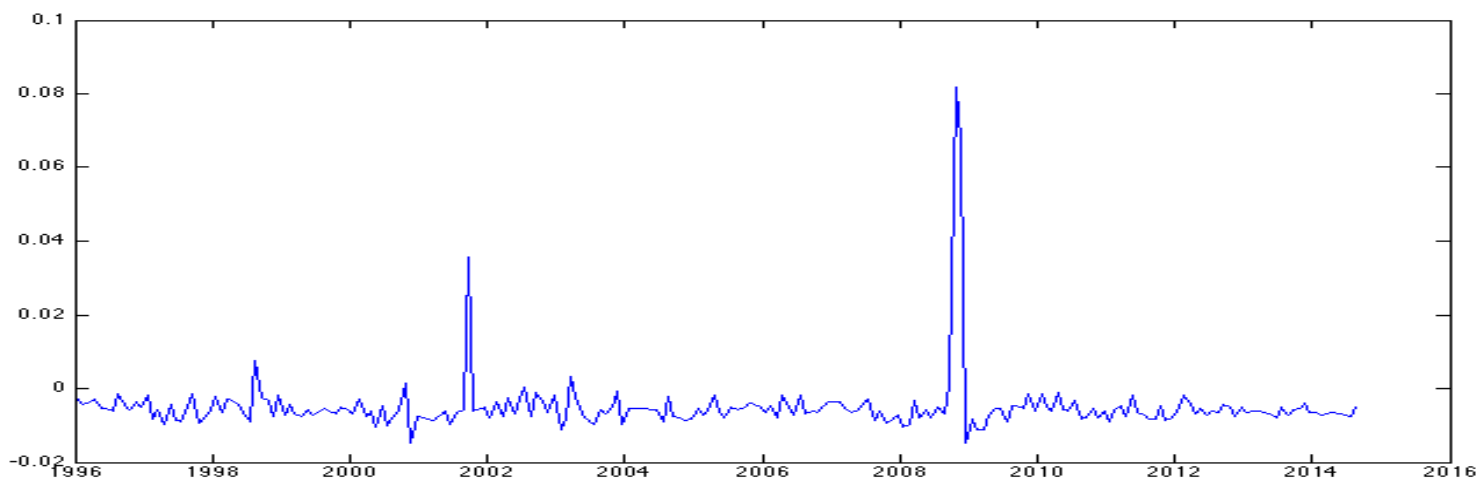

(c) BSIV and MFIV-EBIV

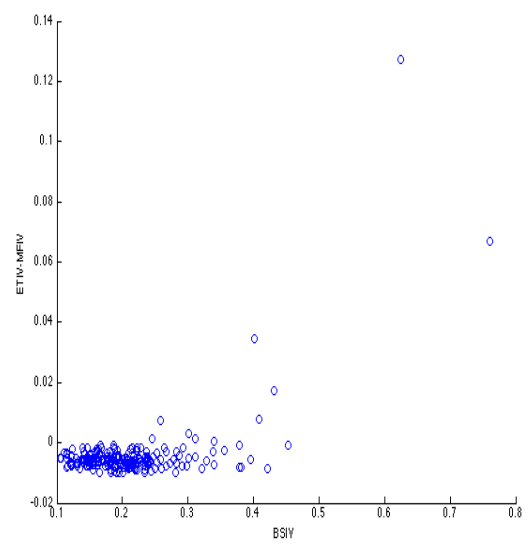

Note: This figure shows the comparison between the MFIV and the EBIV from January 1996 to August 2014. In $2 \mathrm{a}$, the blue and red lines represent the MFIV and the EBIV respectively. Figure $2 \mathrm{~b}$ shows the spread between EBIV and MFIV. Figure 2c shows the scatter plot between the BSIV (horizontal axis) and the spread between MFIV and EBIV (vertical axis). 
Figure 3: Confidence interval using the maximum entropy method

(a) Confidence interval for EBIV

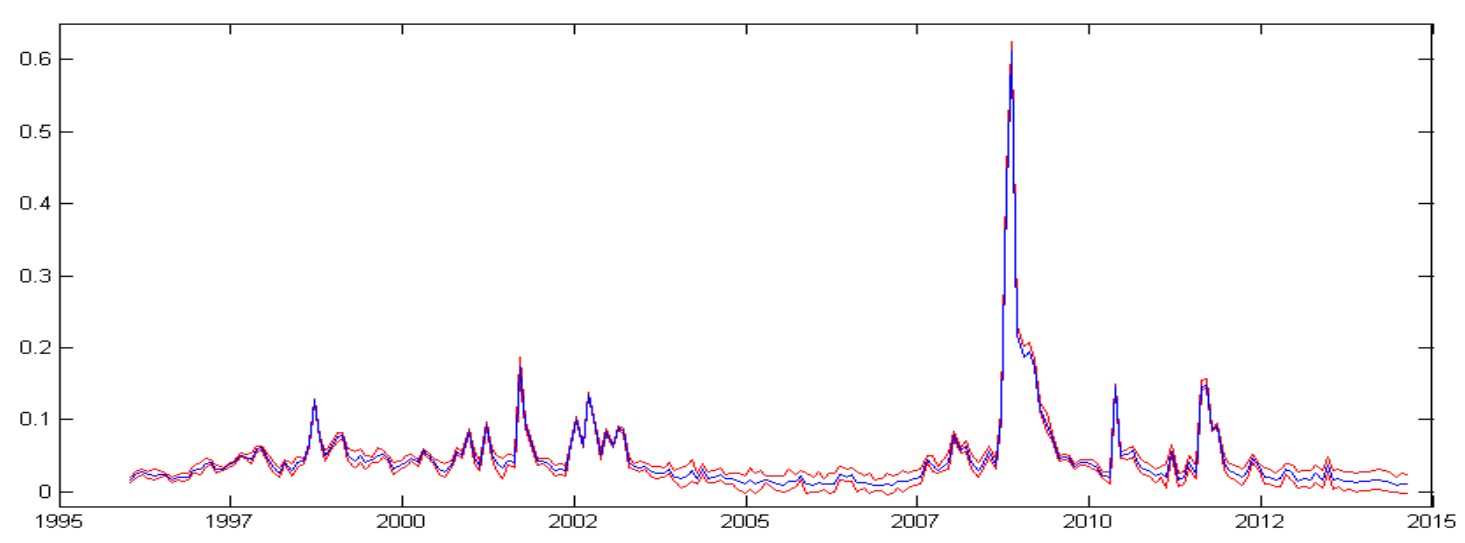

(b) Length of the confidence interval

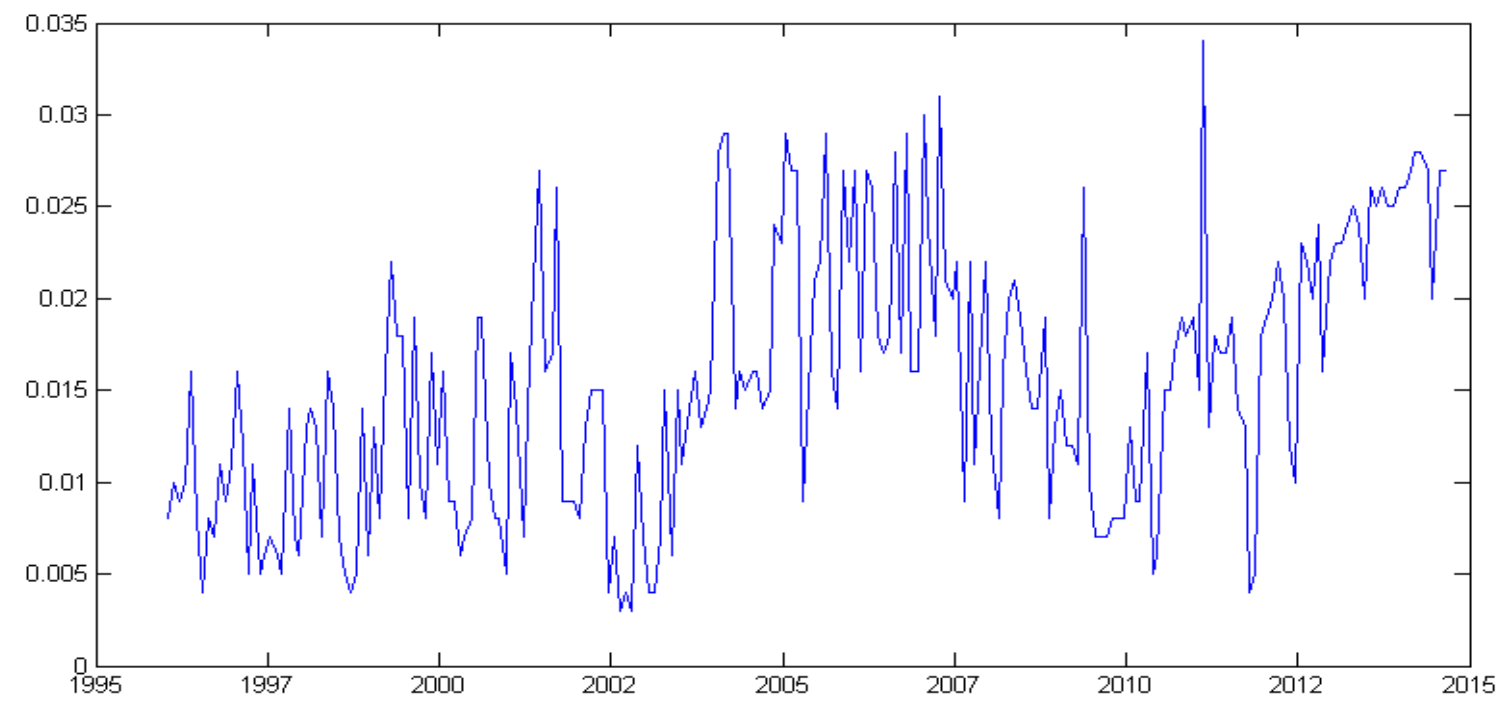

Note: This figure shows the confidence interval obtained from the maximum entropy method. In Figure 3a, the red lines show the upper and lower bounds of the $95 \%$ confidence interval around the point estimate of the EBIV (indicated by the blue line). Figure $3 \mathrm{~b}$ shows the time series of the lengths of the confidence intervals. 
Figure 4: Estimated risk neutral distributions on four selected dates

(a) June 18th, 1998

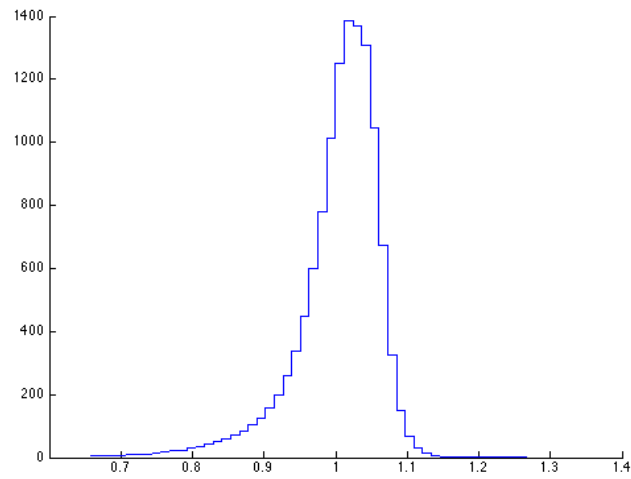

(c) October, 23rd, 2008

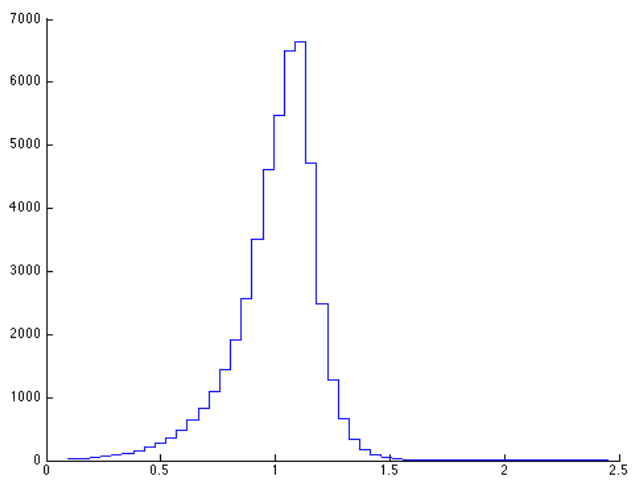

(b) September 20th, 2001

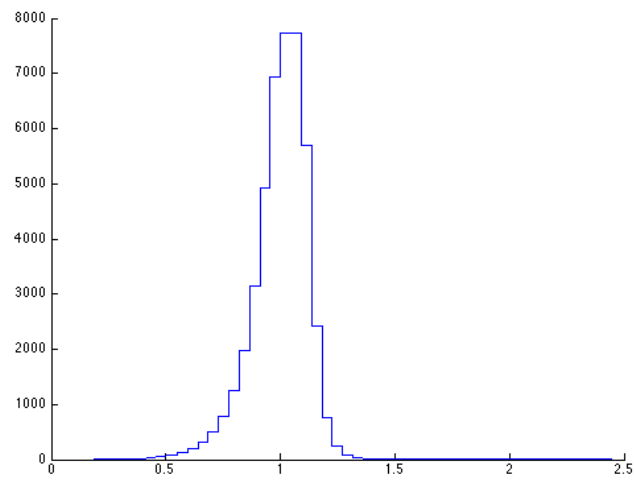

(d) June 19th, 2014

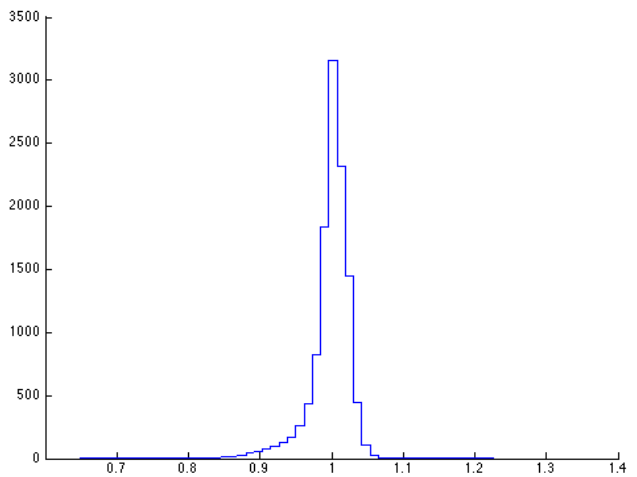

Note: This figure shows the estimated risk neutral distributions using the maximum entropy method on four selected dates. 


\section{References}

Aït-Sahalia, Y., Lo, A. W., 1998. Nonparametric estimation of state-price densities implicit in financial asset prices. Journal of Finance 53, 499-547.

Andersen, T. G., Bondarenko, O., Gonzalez-Perez, M. T., 2015. Exploring return dynamics via corridor implied volatility. Review of Financial Studies p. hhv033.

Bakshi, G., Cao, C., Chen, Z., 1997. Empirical performance of alternative option pricing models. Journal of Finance 52, 2003-2049.

Bakshi, G., Madan, D., 2000. Spanning and derivative-security valuation. Journal of financial economics 55, 205-238.

Bakshi, G. S., Kapadia, N., Madan, D., 2003. Stock return characteristics, skew laws, and the differential pricing of individual equity options. Review of Financial Studies 16, 101-143.

Bali, T. G., Murray, S., 2013. Does risk-neutral skewness predict the cross-section of equity option portfolio returns? Journal of Financial and Quantitative Analysis 48, 1145-1171.

Bates, D. S., 1991. The crash of' 87 : Was it expected? the evidence from options markets. Journal of Finance 46, 1009-1044.

Black, F., Scholes, M., 1973. The pricing of options and corporate liabilities. Journal of Political Economy pp. 637-654.

Blair, B. J., Poon, S. H., Taylor, S. J., 2001. Modelling S\&P 100 volatility: The information content of stock returns. Journal of Banking and Finance 25, 1665-1679.

Bollerslev, T., Tauchen, G., Zhou, H., 2009. Expected stock returns and variance risk premia. Review of Financial studies 22, 4463-4492.

Britten-Jones, M., Neuberger, A., 2000. Option prices, implied price processes, and stochastic volatility. The Journal of Finance 55, 839-866.

Buchen, P. W., Kelly, M., 1996. The maximum entropy distribution of an asset inferred from option prices. Journal of Financial and Quantitative Analysis 31, 143-159. 
Busch, T., Christensen, B. J., Nielsen, M. Ø., 2011. The role of implied volatility in forecasting future realized volatility and jumps in foreign exchange, stock, and bond markets. Journal of Econometrics 160, 48-57.

Canina, L., Figlewski, S., 1993. The informational content of implied volatility. Review of Financial studies 6, 659-681.

Carr, P., Lee, R., Wu, L., 2012. Variance swaps on time-changed lévy processes. Finance and Stochastics 16, 335-355.

Carr, P., Wu, L., 2009. Variance risk premiums. Review of Financial Studies 22, 1311-1341.

Christensen, B. J., Hansen, C. S., Prabhala, N. R., 2001. The telescoping overlap problem in options data. Working Paper .

Christensen, B. J., Prabhala, N. R., 1998. The relation between implied and realized volatility. Journal of Financial Economics 50, 125-150.

Clark, T. E., West, K. D., 2007. Approximately normal tests for equal predictive accuracy in nested models. Journal of econometrics 138, 291-311.

Day, T. E., Lewis, C. M., 1992. Stock market volatility and the information content of stock index options. Journal of Econometrics 52, 267-287.

DeMiguel, V., Plyakha, Y., Uppal, R., Vilkov, G., 2014. Improving portfolio selection using option-implied volatility and skewness. Journal of Financial and Quantitative Analysis 48, $1-57$.

Dennis, P., Mayhew, S., 2002. Risk-neutral skewness: Evidence from stock options. Journal of Financial and Quantitative Analysis 37, 471-493.

Diebold, F. X., Mariano, R. S., 1995. Comparing predictive accuracy. Journal of Business \& economic statistics 13, 253-263.

Fleming, J., 1998. The quality of market volatility forecasts implied by s\&p 100 index option prices. Journal of empirical finance 5, 317-345. 
Hansen, B. E., 1994. Autoregressive conditional density estimation. International Economic Review pp. 705-730.

Jackwerth, J. C., 2000. Recovering risk aversion from option prices and realized returns. Review of Financial Studies 13, 433-451.

Jiang, G. J., Tian, Y. S., 2005. The model-free implied volatility and its information content. Review of Financial Studies 18, 1305-1342.

Kitamura, Y., Stutzer, M., 1997. An information-theoretic alternative to generalized method of moments estimation. Econometrica 65, 861-874.

Lamoureux, C. G., Lastrapes, W. D., 1993. Forecasting stock-return variance: Toward an understanding of stochastic implied volatilities. Review of Financial Studies 6, 293-326.

Martin, I., Forthcoming. What is the expected return on the market? Quarterly Journal of Economics .

Neumann, M., Skiadopoulos, G., 2013. Predictable dynamics in higher-order risk-neutral moments: Evidence from the s\&p 500 options. Journal of Financial and Quantitative Analysis 48, 947-977.

Patton, A. J., 2011. Volatility forecast comparison using imperfect volatility proxies. Journal of Econometrics 160, 246-256.

Schennach, S. M., 2007. Point estimation with exponentially tilted empirical likelihood. The Annals of Statistics 35, 634-672.

Stutzer, M., 1996. A simple nonparametric approach to derivative security valuation. The Journal of Finance 51, 1633-1652.

West, K. D., 1996. Asymptotic inference about predictive ability. Econometrica: Journal of the Econometric Society pp. 1067-1084. 